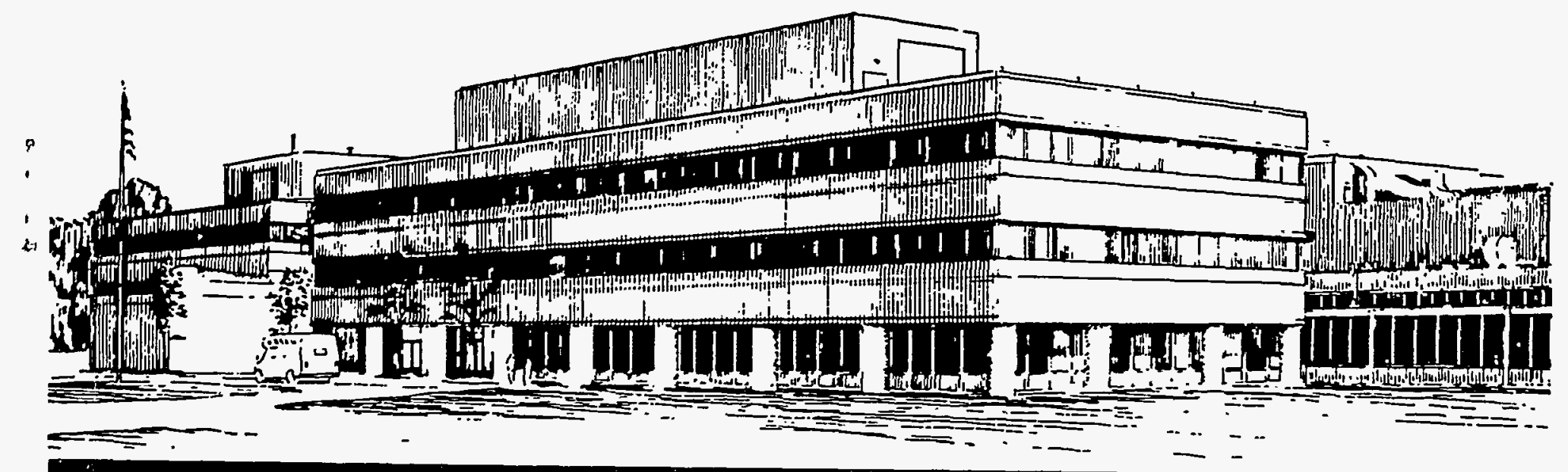

PRINCETON UNIVERSITY, PRINCETON, NEW JERSEY 


\section{NOTICE}

This report was prepared as an account of work sponsored by an agency of the United States Government. Neither the United States Government nor any agency thereof, nor any of their employees, makes any warranty, express or implied, or assumes any legal liability or responsibility for the accuracy, completeness, or usefulness of any information, apparatus, product, or process disclosed, or represents that its use would not infringe privately owned rights. Reference herein to any specific commercial produce, process, or service by trade name, trademark, manufacturer, or otherwise, does not necessarily constitute or imply its endorsement, recommendation, or favoring by the United States Government or any agency thereof. The views and opinions of authors expressed herein do not necessarily state or reflect those of the United States Government or any agency thereof.

\section{NOTICE}

This report has been reproduced from the best available copy. Available in paper copy and microfiche.

Number of pages in this report: 46

DOE and DOE contractors can obtain copies of this report from:

Office of Scientific and Technical Information P.O. Box 62

Oak Ridge, TN 37831;

(615) 576-8401.

This report is publicly available from the:

National Technical Information Service

Department of Commerce

5285 Port Royal Road

Springfield, Virginia 22161

(703) $487-4650$ 


\section{DISCLAIMER}

Portions of this document may be illegible in electronic image products. Images are produced from the best available original document. 


\title{
Transport in Gyrokinetic Tokamaks
}

\author{
H. E. Mynick and S. E. Parker \\ Plasma Physics Laboratory, Princeton University \\ P.O. Box 451 \\ Princeton, New Jersey 08543-0451, U.S.A.
}

\begin{abstract}
A comprehensive study of transport in full-volume gyrokinetic (gk) simulations of ion temperature gradient driven turbulence in core tokamak plasmas is presented. Though this "gyrokinetic tokamak" is much simpler than experimental tokamaks, such simplicity is an asset, because a dependable nonlinear transport theory for such systems should be more attainable. Toward this end, we pursue two related lines of inquiry. (1)We study the scalings of gk tokamaks with respect to important system parameters. In contrast to real machines, the scalings of larger gk systems $\left(a / \rho_{s} \gtrsim 64\right)$ with minor radius, with current, and with $a / \rho_{s}$ are roughly consistent with the approximate theoretical expectations for electrostatic turbulent transport which exist as yet. Smaller systems manifest quite different scalings, which aids in interpreting differing mass-scaling results in other work. (2)With the goal of developing a first-principles theory of gk transport, we use the gk data to infer the underlying transport physics. The data indicate that, of the many modes $k$ present in the simulation, only a modest number $\left(N_{k} \sim 10\right)$ of $k$ dominate the transport, and for each, only a handful $\left(N_{p} \sim 5\right)$ of couplings to other modes $p$ appear to be significant, implying that the essential transport physics may be described by a-far simpler system than would have been expected on the basis of earlier nonlinear theory alone. Part of this analysis is the inference of the coupling coefficients $M_{k p q}$ governing the nonlinear mode interactions, whose measurement from tokamak simulation data is presented here for the first time.
\end{abstract}

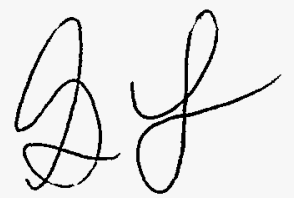




\section{Introduction}

One of the outstanding problems in magnetic fusion research is the development of a theory of tokamak transport which reliably predicts experimentally observed transport levels. It is generally accepted that 'anomalous transport,' i.e., the disparity between observed transport and neoclassical collisional transport, ${ }^{1-3}$ is due to turbulent plasma fluctuations, and since the early '70s a great deal of effort (see, for example, Ref. 4) has been expended to develop a dependable theory of tokamak turbulent transport. Despite some successes, however, this goal has as yet proven elusive, due to limitations on diagnostic information, and to complexities of both the experiment and of turbulent transport theories.

Powerful new tools in this endeavor are gyrokinetic (gk) simulation codes. ${ }^{5}$ These can now simulate a full toroidal volume ${ }^{6}$ with up to a few million particles, and so contain much of the physics of real tokamak experiments. Both the real and numerical 'experiments' are large statistical systems, with very similar particle dynamics, and are therefore capable of supporting a similar set of fluctuations. However, the gk simulations are also far simpler than real experiments - they contain no atomic physics, usually assume adiabatic electrons and a single ion species, support only low-frequency electrostatic perturbations - and are also far simpler to diagnose. Thus, the development of a reliable theory of transport in gyrokinetic tokamaks would represent a sizeable step toward the ultimate goal of such a theory for real tokamaks, but should be more attainable. In this paper, we adopt this goal, using the gk data to guide the development of a transport theory, in testing the validity of assumptions, suggesting satisfactory approximations, etc.

We pursue two related lines of study, each with its counterpart in what has been done for real experiments: (1)studies of the scalings of the transport with important plasma parameters, and (2)studies aimed at obtaining a valid first-principles nonlinear theory describing the transport. Regarding line (1), the gk scalings provide an intermediate 'data point' between experimental scalings and theoretical predictions, giving added insight into the origins of existing theoretical/experimental differences. Addressed here, and touching on many of the current issues of interest in tokamak transport, are the scaling of transport with minor radius $r$, with temperature $T$, with magnetic field strength $B$, with ion mass $M_{i}$, and with safety factor $q$ (or current $I_{p}$ ). And regarding line (2), probably the major obstacle in obtaining a cred- 
ible nonlinear theory is finding the saturated fluctuation amplitudes. For highly idealized problems such as the Hasegawa-Mima ${ }^{7}$ and Terry-Horton ${ }^{8}$ equations, where the linear and nonlinear coefficients $L_{k}$ and $M_{k p q}$ governing the dynamics are known and fairly simple, reliable predictions for the saturated spectrum can be found from numerically-intensive direct simulation $^{9,10}$ or statistical closures. ${ }^{11,12}$ However, for the full kinetic toroidal problem, where accurate evaluation of even the linear coefficients requires extensive numerical computation, the complexity of the coupled nonlinear equations generally results in drastic assumptions or approximations being made, making the resultant transport coefficients rough estimates at best. Here, we show that for gk tokamaks, the data indicate that major simplifications in the full complexity of these equations occur, which should permit a greatly reduced description of the essential transport physics. A major part of these simplifications occurs in the character of the nonlinear coupling coefficients $M_{k p q}$, whose numerical measurement from the gk data (along with the linear coefficients) is presented for the first time in this work.

The paper is organized as follows. In Sec. II we summarize the gk simulation method used, introducing the needed notation and underlying equations, and present some characteristic simulation results. Sec. III begins the analysis of gk transport, discussing the radial scaling of the heat flux $Q$, its harmonic decomposition $Q=\sum_{k} Q_{k}$, time-dependence, and the relation between the $Q_{k}(t)$ and the mode amplitudes $\phi_{k}(t)$. Knowing this relationship, what is additionally required for a complete theory of the fluxes is a description of the $\phi_{k}$ in the saturated steady-state, governed by the nonlinear physics. This is studied in Sec. IV, where the coupling coefficients $M_{k p q}$ are found, and a reduced description of the mode dynamics is distilled. The $M_{k p q}$ are used to infer the energy transfer among the modes in the reduced description. Global scalings are investigated in Sec. V, where the gk-scalings with $T, B, M$, and $q$ are obtained, and compared with analytic and experimental scalings. A discussion summarizing the findings of the earlier sections is given in Sec. VI.

\section{Gyrokinetic Simulation}

We briefly describe the full-volume toroidal simulation method used for this study. A fuller account has been given earlier. ${ }^{6,13}$ The code uses a fully nonlinear $\delta f$-method ${ }^{13,14}$ in an azimuthally symmetric volume with a square poloidal cross-section, of width $L_{x}$, height $L_{y}=L_{x}$, and with major 
radius $R=R_{0}$ at the center of the box (Fig. 1). The box is parametrized by $\mathrm{x} \equiv(x, y, \zeta)$, with $\zeta$ the toroidal azimuth, and with $x=0=y$ at the magnetic axis. The poloidal plane may also be parametrized by the usual minor radius $r \equiv \sqrt{x^{2}+y^{2}}$ and poloidal azimuth $\theta$. The magnetic field lies on circular surfaces $r=$ constant, with a quadratic model for the safety factor $q(r)=q_{0}+\Delta q(r / a)^{2}$, where $a \simeq L_{x} / 2$ at the plasma edge.

A particle moves in a 6-dimensional phase space $\mathbf{z}$, parametrized by $\mathbf{z} \equiv$ $\left(\mathbf{R}, v_{\|} ; \theta_{g}, \mu\right)$, with guiding-center position $\mathbf{R}$, parallel velocity $v_{\|}$, magnetic moment $\mu \equiv M v_{\perp}^{2} /(2 B)$, and gyrophase $\theta_{g}$. However, as usual, $\theta_{g}$ does not enter the gk equations of motion, so that its conjugate momentum $\mu$ is invariant, and the time-evolution of these two can be suppressed. Assuming electrostatic perturbations only, the evolution of $\mathbf{R}$ and $v_{\|}$are given by ${ }^{15}$

$$
\begin{aligned}
\dot{\mathbf{R}} & =v_{\|} \hat{\mathbf{b}}+\frac{\hat{\mathbf{b}}}{M \Omega_{g}} \times\left(M v_{\|}^{2} \kappa+\mu \nabla B+e \nabla \psi\right), \\
v_{\|} & =-\frac{1}{M}\left(\hat{\mathbf{b}}+\frac{v_{\|}}{\Omega_{g}} \hat{\mathbf{b}} \times \kappa\right) \cdot(\mu \nabla B+e \nabla \psi),
\end{aligned}
$$

with $\hat{\mathbf{b}} \equiv \mathbf{B} / b, \boldsymbol{\kappa} \equiv \hat{\mathbf{b}} \cdot \nabla \hat{\mathbf{b}}$ the field-line curvature, and $\psi \equiv\langle\phi\rangle_{\theta_{g}} \simeq$ $J_{0}\left(k_{\perp} \rho_{g}\right) \phi(\mathbf{R})$ the gyro-averaged electrostatic potential $\phi . \Omega_{g} \equiv e B /(M c)$ is the gyrofrequency, $\rho_{g} \equiv v_{\perp} / \Omega_{g}$ is the gyroradius, and $J_{0}$ is the Bessel function of the first kind.

The Vlasov equation for the evolution of $f\left(\mathbf{R}, v_{\|}, t ; \mu\right)$ is given by

$$
\partial_{t} f+\dot{z} \cdot \partial_{z} f \equiv \partial_{t} f+\dot{\mathbf{R}} \cdot \partial_{\mathbf{R}} f+\dot{v}_{\|} \partial_{v_{\|}} f=0,
$$

where $\partial_{x}$ denotes a partial derivative with respect to any scalar $x$, or if $x$ is vector, a gradient in $x$-space. Both $\dot{z}$ and $f$ may be decomposed into an equilibrium and perturbed part, $\dot{\mathbf{z}}=\dot{\mathbf{z}}_{0}+\dot{\mathbf{z}}_{1}$ and $f=f_{0}(\mathbf{z})+\delta f(\mathbf{z}, t)$, where $\dot{\mathbf{z}}_{1}$ are those terms in Eqs. 1 and 2 proportional to $\psi$, and $\dot{\mathbf{z}}_{0}$ are the remainder, involving equilibrium fields only. Eq.(3) then gives equations for $f_{0}$ and $\delta f: \dot{\mathbf{z}}_{0} \cdot \partial_{\mathbf{z}} f_{0}=0$, and

$$
\partial_{t} \delta f+\dot{\mathbf{z}}_{0} \cdot \partial_{\mathbf{z}} \delta f+\dot{\mathbf{z}}_{1} \cdot \partial_{\mathbf{z}} \delta f=-\dot{\mathbf{z}}_{1} \cdot \partial_{\mathbf{z}} f_{0} .
$$

In the simulation Eq.(4) is integrated along the characteristics given by Eqs. 1 and 2 to accumulate $\delta f=\sum_{i} w_{i} \delta\left(\mathbf{z}-\mathbf{z}_{i}\right)$ or, more precisely, the weight $w_{i}$ of the $i^{\text {th }}$ particle. ${ }^{13} f_{0}$ is taken to be a local Maxwellian, with $\partial_{\mathbf{R}} f_{0}=-\hat{\mathbf{r}} \kappa f_{0}$, where $\kappa=\left[\kappa_{n}+\kappa_{T}\left(v^{2} /\left(2 v_{t}^{2}\right)-3 / 2\right)\right], \kappa_{n}(r) \equiv-\partial_{r} \ln n_{0}(r)$, and $\kappa_{T}(r) \equiv-\partial_{r} \ln T(r)$. 
The perturbing fields are obtained from solving the gyrokinetic Poisson's equation:

$$
\lambda_{i}^{-2}\left(1-\Gamma_{0}(b)\right) \phi_{k}=4 \pi\left(e_{i} \bar{n}_{i k}+e_{e} n_{e k}\right),
$$

with $b \equiv\left(k_{\perp} \rho_{g i}\right)^{2}, \Gamma_{0}(b) \equiv I_{0}(b) e^{-b}, \lambda_{i} \equiv\left(T_{i} / 4 \pi n_{i 0} e_{i}^{2}\right)^{1 / 2}$ the ion Debye length, $e_{i} \equiv Z_{i} e_{0}$ the ion charge, $e_{e}=-e_{0}$ electron charge, $n_{e}$ the electron density, and $\bar{n}_{i} \equiv \int d \mathbf{v} J_{0} f_{i}(\mathbf{z})$ the density of ion guiding-centers. Only a single species of ions is followed, and the electron response is taken equal to 0 for $(m, n)=0$, and otherwise as adiabatic: $n_{e k}=e_{0} \phi_{k} / T_{e}$. ( $m$ and $n$ are the poloidal and toroidal harmonics.) Hence, effects of self-generated shear flow are not included in this model, ${ }^{16-18}$ nor are trapped electron, nor electromagnetic effects. The system is essentially able to support only electrostatic ion temperature gradient-driven instabilities, a useful simplification for purposes of analysis.

Some characteristic simulation results are shown in Figs. 2-4. In gk units $\left[x \rightarrow x / \rho_{s}, t \rightarrow \Omega_{g i} t, E \rightarrow E / T_{e}\right]$ (for any energy $E$ ), the parameters used are $R_{0}=892, a=64, q_{0}=1.25, \Delta q=3, \tau \equiv T_{e} / T_{i}=1$, with with $\kappa_{n}=0$, and temperature profile $T=T_{0} \exp \left[-\kappa_{T 0} L_{T 1} \tanh \left(\left(r-r_{0}\right) / L_{T 1}\right)\right]$, with $\kappa_{T 0}=$ $1 / 50, L_{T 1}=20, r_{0}=a / 2$. [Thus, $\kappa_{T}(r)=\kappa_{T 0} \cosh ^{-2}\left(\left(r-r_{0}\right) / L_{T 1}\right)$, which provides the drive for the ion temperature gradient (ITG) instability, peaks at $r=r_{0}$.] In Fig. 2 is shown the radially-averaged ion heat flux $\bar{Q}_{i}^{r}$ versus time. One notes three phases characteristic of these simulations:(a)an initial linear growth phase, (b)a phase where $\bar{Q}^{r}$ nonlinearly saturates and begins to fall, and (c)a final quasisteady saturated state. Phase-c is the most relevant to real experiments. Figures $3(\mathrm{a}, \mathrm{b})$ show contours of constant potential in linear growth phase-a, in poloidal cross-section (a), and in the midplane (b). Phase-a shows the typical character of the linear modes: radially global, peaking near radius $r=r_{0}$, with fixed $n$ (in this case, $n=4$ ), and with a ballooning character, largest around $\theta=0$, and with the dominant $m$ changing as one moves radially outward, to satisfy $k_{\|}=(n+m / q(r)) / R \simeq 0$. Fourier transforming $\phi$ over a flux surface, one finds the mode typically dominated one value of $m$ (and its complex conjugate), sometimes plus one or two subdominant sidebands, as shown in Fig. 3(c). Thus, labelling the linear modes by mode index $a$, one may designate $a$ by the approximate assignment $a \rightarrow[n, m(r) \simeq-q(r) n]$, for some chosen flux surface $r$, which we choose to be $r=r_{0}$.

In phase-c (Figs. 4), other modes have grown up, breaking up the coherent structure of phase-a, but leaving the characteristic scales in both the radial and poloidal directions qualitatively similar. 


\section{Transport}

For a given $r$, the flux-surface averaged heat-flux for species $s Q_{s}(r, t)$ may be written

$$
\begin{aligned}
Q_{s} & =V_{r}^{-1} \int_{V_{r}} d \mathbf{z} \dot{r}_{E} \delta f_{s}\left(M v^{2} / 2\right)=V_{r}^{-1} \int_{V_{r}} d \mathbf{R} \dot{r}_{E} \delta p_{s} \\
& =\sum_{\mathbf{k}} Q_{s k},
\end{aligned}
$$

where $Q_{k}=i\left(c k_{\theta} / B\right) \phi_{-k} p_{k} / N_{f}^{2}$ is the contribution of harmonic $\mathbf{k}$ to $Q$, $\mathbf{k} \equiv\left(k_{\theta}=m / r, k_{\zeta}=n / R_{0}\right), \delta p(r, \theta, \zeta)=N_{f}^{-1} \sum_{\mathbf{k}} p_{k}(r) \exp i(m \theta+n \zeta)$ is the perturbed pressure, $\dot{r}_{E} \equiv \hat{\mathbf{r}} \cdot \dot{\mathbf{R}}_{1}=\hat{\mathbf{r}} \cdot(c / B) \hat{\mathbf{b}} \times \nabla \psi$ is the $E \times B$-portion of the radial drift velocity, $N_{f}$ is the number of mesh-points taken over a flux surface, and $\int_{V_{\tau}}(\ldots)$ denotes an integral over a thin toroidal shell $V_{r}$ centered at $r$. An analogous expression holds for the particle flux $\Gamma_{s}$, but with the replacements $M v^{2} / 2 \rightarrow 1, \delta p \rightarrow \delta n$, and $p_{k} \rightarrow n_{k}$ above. The gk results for the fluxes are quite similar whether computed from the fluidlike expressions given above, with $\delta p$ or $\delta n$ first accumulated at each mesh point, or from the kinetic expression using a direct summation over all particles within shell $V_{r}$. Using the approximate assignment $a \rightarrow \mathrm{k}\left(r=r_{0}\right)$ for mode label noted in the previous section, one may replace $\sum_{\mathbf{k}}$ in the final form in Eq.(6) with a summation $\sum_{a}$ over contributions from each mode $a$.

We first consider the $r$-variation of $Q$. For real experiments, it is wellknown ${ }^{19}$ that $\chi(r)$ continues to rise as one moves out toward $r=a$, in conflict with both the Bohm expression $\chi_{B}=c_{B} \rho_{s} c_{s}$, and the gyroBohm (gB) estimate $\chi_{g B} \equiv c_{g B} \kappa_{T} \rho_{s}^{2} c_{s}$ which arises from drift-wave theories. (Here, $c_{B}$ and $c_{g B}$ are numerical factors, whose value is fixed by nonlinear physics.) For these, the positive dependence on $T(r)\left(\chi_{B} \sim T\right.$ and $\left.\chi_{g B} \sim T^{3 / 2}\right)$ causes $\chi(r)$ or $Q(r)=-\chi n \partial_{r} T(r)$ to fall off as $r \rightarrow a$, as shown in Figs. 5 (drawn for $c_{B}=1=c_{g B}$ for the profiles used in the simulations.) Contrasting with experimental results is the strong resemblance of $Q_{B}$ and $Q_{g B}$ to the timeaveraged result $\bar{Q}_{g k}^{t}(r)$ from the gk simulation plotted in Fig. 6. However, the reason for the radial dependence here is somewhat different from that for standard drift wave theories for real tokamaks, which typically assume radially localized perturbations. For these, mixing-length estimates assume $\chi \sim \gamma / k_{r}^{2}$, with $\gamma \sim \omega_{*}^{f} \equiv-k_{\theta} \kappa c T /(e B)$. Thus, $\chi \sim \chi_{B}\left(\kappa k_{\theta} / k_{r}^{2}\right)$, with $k_{\theta} \sim \rho_{g}^{-1}$ and the replacements $k_{r} \rightarrow k_{\theta}$ for $\chi_{g B}$ and $k_{r}^{2} \rightarrow k_{\theta} \kappa$ for $\chi_{B}$. The falloff toward the edge in this standard argument is thus due to a corresponding falloff in the $\gamma / k_{\perp}^{2}$ of the individual localized modes as $r$ increases. 
In contrast, for each radially global mode $a$ dominating gk transport, one has a single $\gamma_{a}$, but a radially-varying mode amplitude, giving a similar radial dependence to $Q_{a}(r)$ in Eq.(6).

The global character of the modes implies that knowing their evolution at $r=r_{0}$ specifies their evolution, and their effect on transport, at all $r$. Thus, henceforth we look at the $Q_{k}$ at $r=r_{0}$. We consider ion transport, suppressing species label $i$. We first rank the $Q_{k}$ at $r=r_{0}, Q_{k_{1}} \geq Q_{k_{2}} \geq$ $Q_{k_{3}} \geq \ldots$, and in Fig. 7 plot the running sum $Q_{j} \equiv \sum_{i=1}^{j} Q_{k_{i}}$. One notes that, of the thousands of $k$ 's which could in principle contribute significantly to transport, in fact only a modest number $N_{k} \sim 10$ of modes account for most of the full flux $Q=Q_{j \rightarrow \infty}$. Taking $N_{k}=12\left(6 \phi_{k}\right.$ plus their complex conjugates, which contribute equally to the flux), in Fig. 8 we plot (a)the largest $Q_{k}^{g k}$ from the gk data versus time, and (b) the corresponding $\left|\phi_{k}\right|(t)$.

The radial dependence having been dealt with, for a complete description of the fluxes one needs two additional ingredients:(1) given the spectrum $\left\{\phi_{k}\right\}$, an expression for the corresponding $\left(n_{k}, p_{k}\right)$ to use in Eq.(6), and (2) a specification of the saturated steady-state spectrum $\left\{\phi_{k}\right\}$ corresponding to phase-c of Fig. 2. The latter requires an understanding of the nonlinear physics, discussed in Sec. IV. The simplest and most widely-used specification for (1) is the quasilinear flux $\Gamma^{q l}$ or $Q^{q l}$, obtained from using the linear response for $\delta f$ in Eq.(6). This yields $n_{s k} \equiv-\left(e_{s} \phi_{k} / T_{s}\right) n_{s 0} g_{s k}$ and $p_{s k} \equiv-\left(e_{s} \phi_{k} / T_{s}\right) p_{s 0} q_{s k}$, with $g_{s k}(\omega) \equiv k^{2} \lambda_{s}^{2} K_{s k}(\omega)$ the linear-response function for the density [ $K_{s k}(\omega)$ is the linear susceptibility], and $q_{s k}(\omega)$ the linear-response function for the pressure. For adiabatic electrons $g_{e k}=1$. Expressions for $g_{i k}$ and $q_{i k}$ are given in Appendix A. Using these, one has, from Eq.(6)

$$
e_{s}\left[\begin{array}{c}
\Gamma_{s k} \\
Q_{s k} / T_{s}
\end{array}\right]^{q l}=-i \frac{c k_{\theta}}{B} \frac{e_{s}^{2} n_{s o}}{T_{s}}\left|\phi_{k}\right|^{2}\left[\begin{array}{c}
g_{s k} \\
q_{s k}
\end{array}\right](\omega) .
$$

With the approximate evaluation of these sketched in App. A, in Fig. 8c we plot the quasilinear fluxes $Q_{k}^{g l}$ using the $\phi_{k}$ in Fig. 8b. Comparing these with Fig. 8a, one sees that the time-dependence of the fluxes $Q_{k}^{g k}$ is consistent with the quasilinear expectation $Q_{k}(t) \sim\left|\phi_{k}\right|^{2}(t)$. The numerical disparity $Q_{k}^{q l} / Q_{k}^{g k} \sim 4$ here is unsurprising, as the evaluation of $g_{i k}$ and $q_{i k}$ used is rather simplified, and is consistent with a parallel disparity in the ratio $\gamma^{a n} / \gamma^{g k} \sim 3$ of the analytic to the numerically-found growth rates. Moreover, as discussed in App. A, while useful for purposes of estimation, 
quasilinear theory omits from the fluxes terms involving the nonlinear response which in the nonlinear phases may be comparable to the linear terms kept.

\section{Nonlinear Physics}

As noted in Sec. I, the greatest difficulty in the development of a credible transport theory lies in understanding the nonlinear physics, which determines the saturation amplitudes of the $\phi_{k}$. In this section we investigate what the gk data tell us about this.

We assume that the mode interactions can be described keeping only up to a quadratic nonlinearity:

$$
\partial_{t} \phi_{k}=L_{k} \phi_{k}+\frac{1}{2} \sum_{0=k+p+q} M_{k p q} \phi_{p}^{*} \phi_{q}^{*}
$$

with $L_{k} \equiv-i \omega_{k} \equiv\left(\gamma_{k}-i \omega_{r k}\right)$ the coefficients characterizing the linear response, and with $M_{k p q}=M_{k q p}$ the nonlinear coupling coefficients. Eq.(8) may be used to describe both weak and some strong turbulent dynamics. As illustrated in Fig. 9 for $n=2$, in the nonlinear phase-c of the simulations, the half-width $\delta \omega$ of the power-spectrum of $\phi(r, \theta, n)$ is a moderate fraction of $\omega$. This, along with the aderence to the quadratic dependence $Q_{k}(t) \sim$ $\left|\phi_{k}\right|^{2}(t)$ observed in Sec. III, suggests that a weak-turbulence treatment may be adequate ${ }^{20}$ though perhaps only marginally. From Eq.(8), one notes that, in addition to the modes $k=\left(\mathbf{k}, \omega_{k}\right)$ which dominantly contribute to transport, there may be modes $p$ (or $q$ ) which, while their contributions $Q_{p}$ to $Q$ may be small, are strongly coupled to some $k$, and are therefore crucial in determining the saturation level of $k$, and so must be retained in a description of the essential transport physics.

As shown in App. A, an analytic expression for the $M_{k p q}$ may be obtained from a weak turbulence treatment. Even with simplifications from employing a local treatment and dropping sideband bounce-harmonics, the general formal expression shown there is complicated, one example of the difficulties encountered in obtaining a reliable analytic nonlinear theory. Accordingly, here we are mainly interested in what the gk simulations reveal about the nonlinear couplings, which make no such simplifications. Techniques have been developed ${ }^{21,22}$ for inferring the strength of mode-coupling in real experiments. Here, we extend the method of Ritz, et al. ${ }^{21}$ to infer both the $L_{k}$ and $M_{k p q}$ for the gk experiment. The method requires as input 
simply an ensemble of $N_{K}$ pairs $\left\{\phi_{k}(t), \phi_{k}\left(t+\tau_{1}\right)\right\}$ of harmonic amplitudes evaluated at 2 nearby times, and so may be applied rather generally, not just to real experiments or the results of the gk code used here, but to any other sort of simulation codes (e.g., field-line following gk codes, gyrofluid codes, or MHD codes) which can produce an analogous set of data. The method is summarized in App. B.

Since our objective is to use the gk data to obtain a reduced description containing the essential transport physics, we need in addition a procedure for selecting which modes $k$ to retain, to which the method of Ref. 21 will be applied. The approach used here is somewhat reminiscent of that used by Aubrey, et al. in obtaining a reduced description of 1-D Navier-Stokes turbulent flow near a wall. ${ }^{23}$ Step 1 of the selection process has already been indicated: by the $Q_{k}$-ranking, we choose $N_{k}$ modes which account for most of the transport. For step 2, we wish to determine, for each such $k$, the $N_{p}$ strongest couplings to other modes $p$ (some of which may be among the original $k$ ). Multiplying Eq.(8) by $\phi_{k}^{*}$ and adding its complex conjugate, one obtains an equation for the spectral intensity $B_{k} \equiv\left\langle\left|\phi_{k}\right|^{2}\right\rangle(\langle\ldots\rangle$ denotes ensemble average):

$$
\partial_{t} B_{k}=2 \gamma_{k} B_{k}+\sum_{0=k+p+q} \operatorname{Re}\left(M_{k p q} T_{k p q}^{*}\right)
$$

where $T_{k p q} \equiv\left\langle\phi_{k} \phi_{p} \phi_{q}\right\rangle$ is the (one-time) triplet correlation function, and the 'power transfer function' ${ }^{\prime 21} T(k, p, q) \equiv \operatorname{Re}\left(M_{k p q} T_{k p q}^{*}\right)$ describes the rate of production of $B_{k}$ due to nonlinear coupling to modes $p$ and $q$. From this, one expects that those modes most strongly coupled to mode $k$ will have strong correlations $T_{k p q}$. Therefore, for each of the initial $N_{k}$ modes $k$, we compute $T_{k p q}$ for all $p$ (and so $q$ ) in the simulation, and choose those $N_{p}$ harmonics $p$ with the largest $\left|T_{k p q}\right|$. (Since $T_{k p q}=T_{k q p}, N_{p}$ is always taken as even.) Finally, to the union of all $k$ and $p$ chosen in steps 1 and 2, comprising a total of $N_{K}$ modes in our reduced system, we apply the method of Ref. 21 to infer the system dynamics, described by the coefficients $\left\{L_{k}, M_{k p q}\right\}$. If there were no overlap of each mode chosen by this process, one would have $N_{K}=N_{k}\left(1+N_{p}\right)$. In fact, as will be seen, for the gk simulations there is a great deal of overlap (i.e., many modes $p$ are among the initial $N_{k}$, as well as among the $N_{p}$ for other modes $k$ ), so that $N_{K}$ is in general much smaller than this. For the gk example to be discussed shortly, one has $N_{k}=12, N_{p}=6$, for a maximum possible $N_{K}$ of 84 , but an actual $N_{K}=32$, half of which are complex conjugates of the others. 
We first benchmark the method on an ensemble of $N_{r l}$ realizations of test data $\left\{\phi_{k}(t), \phi_{k}\left(t+\tau_{1}\right)\right\}$ produced from integrating $N_{K}$ o.d.e.'s of the form (8), where the coefficients $L_{k}, M_{k p q}$ have a known numerical value. The initial conditions of the realizations of the ensemble have Gaussian statistics. We take the $L_{k}=\left(\gamma_{k}-i \omega_{r k}\right)$ to have $\gamma_{k}=0$ and real frequency $\omega_{r k}$ in the range expected for the gk data, but otherwise arbitrarily selected (Fig. 10a). The $M_{k p q}$ (real part plotted in Fig. 10b) are chosen to satisfy the constraints

$$
M_{k p q}=M_{k q p}, M_{k p q}^{*}=M_{-k,-p,-q},
$$

and

$$
0=\sigma_{k} M_{k p q}+\sigma_{p} M_{p q k}+\sigma_{q} M_{q k p},
$$

and are otherwise arbitrary. The first of Eqs. 10 may be taken without loss of generality, and the second must hold for real perturbations $\phi(x)$. Eq.(11) implies that the nonlinear term in (8) conserves $W \equiv \sum_{k} \sigma_{k} B_{k}$, for any set of coefficients $\sigma_{k}$. We take the coefficients $\sigma_{k}=1$ for all modes $k$. For Fig. 10 we use a system of $N_{K}=12$ modes, with a 1-dimensional $k$, equal to $\pm 1, \pm 2, \ldots \pm 6$, and the couplings satisfying the $1 \mathrm{D}$ 'triangle identity' $0=k+p+q$, as must the real data. The much smaller number of modes than used in the benchmark of Ref. 21 parallels a difference between the data from experiments and the gk simulation.

In Fig. 11a,b are shown the results inferred by the method for $N_{r l}=$ 2000. Comparing with Fig. 10a,b, one notes that the agreement is quite good. In Fig. 11c-h we show the effect of reducing $N_{r l}$ through 100, 30, and finally to 5 . The real coefficients are fairly well-represented by the inferred values down to $N_{r l}=30$. Remarkably, even at $N_{r l}=5$ the rough features of the real values are reflected in the inferred ones. This robustness is significant, because while it not difficult to collect experimental data over a time long enough to yield a large ensemble, gk ensembles with $N_{r l}$ greater than about 100 becomes computationally prohibitive. A replacement of the ensemble-average with a time-average over an orbit integration of comparable length to the gk data also infers the $L_{k}$ and $M_{k p q}$ with comparable success to the results in Fig. 11.

We now apply the method to the real gk data. The ensemble-average is replaced by a time average within phase-c, with $N_{r l}=240$ samples $\left(t_{i}, t_{i}+\right.$ $\left.\tau_{1}\right)$ spaced $T_{1}=10 \tau_{0}$ apart, $\left(\tau_{0} \equiv 20\right.$ is the simulation time step), and with $\tau_{1}=\tau_{0}$, which is small compared with a decorrelation time $\tau_{c}$, as discussed in App. B. The run is similar to that shown in Fig. 2, but with a much longer phase-c. In Fig. 12, we first display $\left|T_{k p q}\right|$ for a typical dominant $\mathrm{k}$ 
[here, $(m, n)=(-9,4)$ ] over the $\mathbf{p}=\left(m_{2}, n_{2}\right)$-plane. One notes first that, as already indicated for the dominant $\left|\phi_{k}\right|$, the $\left|T_{k p q}\right|$ have appreciable value only near the line $k_{\|}=0$, i.e., the $\mathbf{p}$ (and so $\mathbf{q}$ ) are nearly collinear with k. This might be expected, since harmonics far from the $k_{\|}=0$ line will be strongly damped, and hence will produce small values of $\left|T_{k p q}\right| \sim \phi_{k} \phi_{p} \phi_{q}$. And second, and probably more surprising, even along this roughly $1 \mathrm{D}$ locus in the 2D p-plane, the $\left|T_{k p q}\right|$ are dominated by only a handful $\left(N_{p} \sim 5\right)$ of p. This enormously simplifies the complexity represented by the nonlinear term in Eq. (8).

Using these $\left|T_{k p q}\right|$ to select the $N_{K}$ modes in the reduced system, and applying to these modes the method of Ritz, et al. yields the results in Fig. 13. In Fig. 13a, $I m L_{k}=-\omega_{\tau k}$ is roughly linear with $n \propto k_{\perp}$, about as one expects for drift-type modes, and Fig. 13b shows a region of modes $R e L_{k}=\gamma_{k}>0$ most unstable for small $|n| \sim 3$, with the maximum $\gamma$ falling as one moves toward higher $|n|$ and towards $n=0$, as expected from the ITG analytic theory. The $M_{k p q}$ plotted in Fig. $13 \mathrm{c}$ have the same characteristics as discussed for the $T_{k p q}$ : appreciable for only a small number of $\mathrm{p}$ 's, lying nearly collinear with $\mathbf{k}$.

The mode-selection process is fairly robust. In the results shown, the initial $N_{k}=12$ modes were chosen from a $Q_{k}$ ranking in phase-a. Instead ranking the $Q_{k}$ in phase-c, one finds a different set of $N_{k}$ harmonics (arising from shifts in the dominant modes from nonlinear power transfer), but precisely the same full set of $N_{K}=32$ harmonics when the dominant couplings $p$ are added to these in step 2, and so the same results for the $L \mathrm{~s}$ and $M \mathrm{~s}$. This kind of 'closure' of the mode set describing the essential physics is what one expects when a genuinely reduced description is possible.

Having computed the $M_{k p q}$, in Fig. 14a we display the power transfer $T(k, p, q)$ appearing in Eq.(9). Since $\mathbf{k}=(m, n), \mathbf{p}=\left(m_{2}, n_{2}\right)$, and $\mathbf{q}=\left(m_{3}, n_{3}\right)$ are $2 \mathrm{D}$ vectors, to display this conveniently we sum over the $m$ 's at each $n$, reducing the $4 \mathrm{D}$ space $(\mathbf{k}, \mathbf{p})$ to the $2 \mathrm{D}$ plane $\left(n, n_{2}\right)$ shown in the figure. Since, as we have seen, $\mathbf{k}, \mathbf{p}$, and $\mathbf{q}$ lie on a nearly $1 \mathrm{D}$ region, this reduction doesn't lose much information. At each $n$, one sees peaks occurring in pairs of equal height, corresponding to $n_{2}$ and $n_{3}$. Fig. 14b shows the $p$-summation $\sum_{0=k+p+q} T(k, p, q)$ on the right-hand side of Eq. (9), representing the total nonlinear contribution to $\partial_{t} B_{k}$, versus $n$. For each $n$, the multiple mode numbers sometimes occurring correspond to different values of $m$ for that $n$. One sees positive contributions (energy transfer into that mode $k$ from all others) principally for $|n| \leq 2$ and at least one $m$, and neg- 
ative contributions at larger $|n|$. Thus, for this simulation it appears that energy is transfered principally from modes with $|n| \gtrsim 3$, where $\gamma$ is larger (Fig. 13b) to modes at somewhat smaller $|n|,|n| \sim 1-2$. This is consistent with the resultant power spectrum $B_{k}$, shown in Fig. 14c (summed over $m$, as for Fig. 14a).

\section{Global Scalings}

We now turn to the global scalings exhibited by gk tokamaks. As for their experimental counterparts, these scalings exhibit the 'bottom line' behavior which a first principles theory should predict, and thus serve as an important guide for such theories. As noted in Sec. I, here we consider the scaling of transport with $T, B, M$, and $q$. These scalings bear on several experimental issues of interest, including the power degradation observed in L- and H-modes (related to the scaling with $T$ ), current-scaling (scaling with $q$ ), Bohm-versus-gyroBohm scaling ${ }^{25}$ (scaling with $T, B$, and $M$ ), and the beneficial 'isotope scaling' observed in L- and H-modes ${ }^{26}$ and in TFTR supershots ${ }^{27,28}$ (scaling with $M$ ).

For purposes of discussion, it is useful to consider simple power-law dependences of $\chi_{i}$ on these variables. We first write

$$
\chi \simeq T^{y} F_{\chi}(B, M, q),
$$

for some function $F_{\chi}$. We may further specialize to $F_{\chi} \propto q^{x} B^{z} M^{w}$. Using $\tau_{E} \simeq a^{2} / \chi, q \sim B / I_{p}$, and Eq.(12) to eliminate $T$ in the power-balance relation $^{29} \chi \simeq P /(n T R)$ yields the analytic form for $\tau_{E}$

$$
\begin{aligned}
\tau_{E}^{a n} & \simeq a^{2}(P / R n)^{-y /(y+1)} F_{\chi}^{-1 /(y+1)} \\
& \propto P^{-y /(y+1)} B^{-(z+x) /(y+1)} I_{p}^{x /(y+1)} M^{-w /(y+1)} .
\end{aligned}
$$

For the collisionless systems with electrostatic perturbations simulated here, invariance arguments ${ }^{30}$ constrain the dependence of $\chi$ on these variables: $\chi=\chi_{B} \tilde{\chi}\left(\rho_{g} / a, q ; \tau, ..\right)$, where as in Sec. III $\chi_{B}=\rho_{s} c_{s}=c T_{e} /\left(e_{0} B\right)$ is the Bohm value for $\chi, \tau=T_{e} / T_{i}$, and $\tilde{\chi}$ is the heat conductivity in gk units. The dependences of $\tilde{\chi}$ on $T, B$, and $M$ enter only through $\rho_{g} / a \sim$ $(M T)^{1 / 2} / B$, imposing the constraints $z \rightarrow(1-2 y)$ and $w \rightarrow(y-1)$ on the power-law forms above. One notes that both the Bohm and gyroBohm scalings satisfy these constraints. This leaves only 2 free exponents $x$ and $y$, which we now determine numerically. 
In Fig. 15 is shown the ion heat flux (in gk units) $Q \simeq \kappa_{T 0} \rho_{s} \tilde{\chi}$ versus $q_{a} \equiv q(r=a)$, keeping $q_{0}$ fixed at 1.25 , and for $a / \rho_{g}=64$. Besides the value for the shearless system $\left(q_{a}=1.25\right), Q$ increases with increasing $q_{a}$ (decreasing $I_{p}$ ), with exponent $x \simeq .25$, lying between $\mathrm{L}, \mathrm{H}$-mode and supershot behavior, but much closer to the latter. One also notes some additional oscillatory structure superposed on this average behavior. This may be due to successive major rational surfaces moving in past the surface $r=r_{0}$ as $q_{a}$ is increased, as illustrated in Fig. 16. One sees that one period of the oscillation in Fig. 15 occurs from the crossing of the dashed guideline at $q_{a} \simeq 4.5$ to $q_{a} \simeq 8.5$. These values correspond to the crossing of $r=r_{0}$ by the surfaces $q=2$ and 3 in Fig. 16 .

Fig. 17 shows the scaling of $Q$ with system size $a / \rho_{g}$. One notes the variation is nonmonotonic, dividing local power-law fits into two asymptotic regimes, one for 'small' gyrokinetic systems ( $g k-s: a / \rho_{g}<64$ ), approximately fit by exponent $y=y_{s} \simeq 0.0$, and one for 'large' systems

$\left(g k-l: a / \rho_{g}>64\right)$. For the largest system simulated $\left(a / \rho_{g}=128\right)$, the $Q$-value is taken to occur the same amount of time after the phase-b peak in $Q$ as the other points shown, for each of which a steady-state in $Q$ had been reached. Taking that value one finds $y=y_{l} \simeq 1.0$, very nearly the Bohm value $y_{B}=1$. For the $a / \rho_{g}=128$ system, however, after a short plateau $Q$ continues to fall slowly. Thus, a sizeable error bar must be attached to this point, extending downward from the value shown toward the gyroBohm value $y_{g B}=1.5$. Further indication of the size scaling in the $g k-l$ regime comes from looking at the scaling of the spectral width $\left\langle k_{r} \rho_{g}\right\rangle$ with system size. This is shown in Fig. 18 for $a / \rho_{g}=64$ and 128. From mixing-length arguments (cf. Sec. III) one expects gyroBohm $Q$-scaling for $\left\langle k_{r} \rho_{g}\right\rangle$ independent of $a / \rho_{g}$, and Bohm-scaling for $\left\langle k_{r} \rho_{g}\right\rangle \sim\left(\rho_{g} / a\right)^{1 / 2}$, decreasing with increasing system size. The two spectra in Fig. 18 have ratio $\left\langle k_{r} \rho_{g}\right\rangle(128) /\left\langle k_{\tau} \rho_{g}\right\rangle(64) \simeq .72 \sim\left(\rho_{g} / a\right)^{.47}$, lying between Bohm and gyroBohm, but much closer to the former, consistent with the scalings in Fig. 17.

Table 1 summarizes the scalings just discussed, in an order which is monotonic in each of $y, z$, and $w$ for the analytic and numerical scalings. One notes that the $g k-l$ scaling is rather close to Bohm behavior and (noting the error bar) to gyroBohm, while $g k-s$ is quite different. Thus, large gk systems possess global scalings roughly described by analytic estimates based on assumptions consistent with those made in the simulations (viz., electrostatic fluctuations only, $\tau=1$, and $a / \rho_{g} \gg 1$ ), and, as noted in Sec. III, have a 


\begin{tabular}{l|l|l|l}
\hline scaling & $x, y, z, w$ & $\chi \sim q^{x} T^{y} B^{z} M^{w}$ & $\tau_{E}$ \\
\hline \hline $\begin{array}{l}\text { analytic/numerical: } \\
g k-s\end{array}$ & $.25,0,1.0,-1.0$ & $q^{.25} T^{.0} B^{1 \cdot} M^{-1 .}$ & $P^{.0} B^{-1.25} I_{p}^{.25} M^{1 .}$ \\
\hline Bohm & $0,1,-1,0$ & $q^{0} T^{1} B^{-1} M^{0}$ & $P^{-.5} B^{.5} I_{p}^{0} M^{0}$ \\
\hline$g k-l$ & $.25,1.0,-1.0, .0$ & $q^{.25} T^{1.0} B^{-1.0} M^{.0}$ & $P^{-.5} B^{.4} I_{p}^{1} M^{.0}$ \\
\hline gB & $0,1.5,-2, .5$ & $q^{0} T^{1.5} B^{-2} M^{.5}$ & $P^{-.6} B^{.8} I_{p}^{0} M^{-.2}$ \\
\hline $\begin{array}{l}\text { experimental: } \\
\text { L,H-mode }\end{array}$ & $2,1,-2,-1$ & $q^{2} T^{1} B^{-2} M^{-1}$ & $P^{-.5} B^{0} I_{p}^{1} M^{.5}$ \\
\hline Supershot & $0,0,-.6,-.5$ & $q^{0} T^{0} B^{-.6} M^{-.5}$ & $P^{0} B^{.6} I_{p}^{0} M^{.5}$
\end{tabular}

Table 1: Transport Scalings

radial scaling also consistent with theoretical expectation. Small-gk systems resemble supershots in scaling with $P, I_{p}$ and $M$, but differ strongly in scaling with $B$. This resemblance is probably only coincidental, since supershots have $\tau \gg 1$, and in TFTR one has $a / \rho_{g} \simeq 200$, far beyond the $g k$-s range $a / \rho_{g} \lesssim 64$. Some studies of gk-scaling for systems with $\tau \gg 1$ have been done by Santoro ${ }^{31}$ using the same code as here, and a beneficial effect on transport of increased $\tau$, similar to that seen for supershots, ${ }^{32}$ has been observed. Further studies are needed to clarify the gk-scalings in this regime. In the same work, looking at transport with varying mixtures of deuterium and tritium, a modest inverse scaling of $\chi$ with $M(w<0$ and $|w| \lesssim .5)$ was found, similar to the isotope scaling in real machines. Again, however, rather than reflecting the origin of experimental isotope scaling, this may be due to the value $a / \rho_{g}=64$ used for those runs, putting them near the transition from the small $(w=-1.0)$ to the large $(w=0.0)$ system in Fig. 17.

\section{Discussion}

Concluding, we have seen that, in contrast to the scalings of real experiments, the transport scalings of large gk systems with $P, B, I_{p}, M$, and $r$ are roughly consistent with analytic estimates which are based on the same assumptions as used in the simulation. While perhaps not relevant to large experiments, we also note that there is a second, small-system gk scaling, whose existence should be borne in mind both for finding what new physics may govern it, and also to avoid confusing its properties with those of supershots, which it in several respects resembles. The gk simulations exhibit 
some of the $q$ or current-scaling characterizing $\mathrm{L}$ and $\mathrm{H}$-modes, but much more weakly. It remains to be seen whether stronger $q$-scalings would be found as the simulation parameters are advanced closer to those of real experiments, for example from gk systems with larger $a / \rho_{g}$. The $q$-scaling also manifests an interesting oscillatory structure, which may be related to the proximity of major rational surfaces to the surface of maximum drive.

That the large-system simulations are roughly consistent with analytic estimates does not yet mean they are well understood-Bohm and gyroBohm estimates are different in practical terms, but distinguishing between them theoretically requires a more refined grasp of the nonlinear physics than goes into mixing length arguments, from which either result can be derived. Moreover, where the bounds of the $g k-l$ family of systems lie, within which transport appears non-anomalous, has yet to be determined.

Several of the features of a first-principles theory which could provide these answers have been drawn from the gk data. Transport is dominated by radially global modes, and the scaling of $Q$ with $r$ is what one would expect from such modes. The quadratic dependence $Q_{k}(t) \sim\left|\phi_{k}\right|^{2}(t)$ of the heat flux suggests that the system is weakly turbulent. The fairly narrow power spectra of the $\phi_{k}$ in the nonlinear saturated phase support this picture, though suggesting that the turbulence is not far from levels where a strong turbulence treatment would be needed. Within this framework, we have inferred the linear and nonlinear coefficients determining the mode dynamics in Eq.(8), which are free of assumptions which are normally made in analytic treatments. For these, we find that, at least for the range of gk systems investigated, major simplifications occur from the full complexity which could be represented by Eq.(8). Namely, only a modest number $\left(N_{k} \sim 10\right)$ of modes $k$ account for most of the transport, and each of these is coupled to only a small number $\left(N_{p} \sim 5\right)$ of modes $p$. These together permit one to describe the essential transport physics with a system of only $N_{K} \sim 30$ mode amplitudes (15 complex amplitudes plus complex conjugates), sufficiently simple that its dynamics and steady-state behavior may be amenable to analytic or semi-analytic solution. Calculation of the power transfer function predicts a shift slightly downward in $|n|$ from the value at the maximum growth rate, consistent with the power spectrum observed in moving from phase-a to phase-c.

Two further steps need to be taken to provide the desired theory of gk transport. First, it must be established that the reduced system, governed by the coefficients $\left\{L_{k}, M_{k p q}\right\}$ inferred here, does in fact reproduce the behavior 
exhibited by the full gk simulation. Since small errors in the coefficients can cause spurious sources and sinks of energy, this may not be straightforward. And second, the numerically-inferred form of the nonlinear coefficients must be understood analytically, presumably by an appropriate evaluation of the formal analytic expression (A10) or something like it. Work toward these objectives is underway.

\section{Acknowledgments}

The authors are grateful to J.A. Krommes, W.W. Lee, N. Pomphrey, and G. Rewoldt for informative discussions.

This work supported by U.S.Department of Energy Contract No. DEAC02-76-CHO3073. 


\section{Appendix A. Analytic Development}

Here, we sketch the origin of some analytic results used or cited in Secs. III and IV. We start with Eq.(4) for $\delta f$, which we write as

$$
G^{-1} \delta f=-\dot{\mathbf{z}}_{1} \cdot \partial_{\mathbf{z}} f_{0}-\dot{\mathbf{z}}_{1} \cdot \partial_{\mathbf{z}} \delta f,
$$

where $G^{-1} \equiv \partial_{t}+\dot{\mathbf{z}}_{0} \cdot \partial_{\mathbf{z}}$ is the inverse of the propagator along unperturbed trajectories. In the main text, we have parametrized phase space in terms of the physical variables $\mathrm{z}=\left(\mathbf{R}, v_{\|} ; \theta_{g}, \mu\right)$. For formal manipulations in complex geometries, it is often useful to instead use the action-angle parametrization $^{33} \mathbf{z}=(\boldsymbol{\theta}, \mathbf{J})$, where $\mathbf{J} \equiv\left(J_{g}, J_{b}, J_{\zeta}\right)$ are the invariant actions of the unperturbed motion, with $J_{g} \equiv(M c / e) \mu, J_{b}$ the bounce action, and $J_{\zeta} \equiv p_{\zeta}$ the canonical angular momentum. Conjugate to these are the angle variables $\theta \equiv\left(\theta_{g}, \theta_{b}, \theta_{\zeta}\right)$, with $\theta_{g}$ the gyrophase, $\theta_{b}$ the bounce phase, and $\theta_{\zeta} \equiv \bar{\zeta}$ the bounce/transit-averaged toroidal azimuth $\zeta$. In the absence of the perturbing Hamiltonian $h(\mathbf{z})$, these evolve at the constant frequencies $\dot{\theta}=\Omega \equiv\left(\Omega_{g}, \Omega_{b}, \Omega_{\zeta}\right)$.

We may write any phase function $A(\mathbf{z})$ as the sum of its ensembleaveraged and fluctuating parts, $A=\langle A\rangle+\tilde{A}$. Ensemble-averaging Eq.(A1), one finds an expression for the transport in $\mathrm{J}$-space, from which one may obtain expressions for the radial fluxes such as Eq.(6):

$$
\partial_{t}\langle f\rangle=\partial_{t}\langle\delta f\rangle=-\left\langle\dot{\mathbf{z}}_{1} \cdot \partial_{\mathbf{z}} \delta f\right\rangle=-\partial_{\mathbf{J}} \cdot\left\langle\dot{\mathbf{J}}_{1} \delta f\right\rangle,
$$

where we have used Liouville's theorem $\partial_{\mathrm{z}} \cdot \dot{\mathrm{z}}_{0,1}=0$ and the fact that the ensemble-average includes an average $\langle\ldots\rangle_{\theta}$ over the phases, so that $\left\langle\partial_{\boldsymbol{\theta}} \cdot\left(\dot{\boldsymbol{\theta}}_{0,1} A\right)\right\rangle=0$.

To evaluate the action-space flux $\mathbf{\Gamma} \equiv\left\langle\dot{\mathbf{J}}_{1} \delta f\right\rangle$ on the right side of Eq.(A2), we formally solve (A1) for $\delta f$, and insert this into the expression for $\Gamma$ :

$$
\Gamma=\Gamma^{q l}+\Gamma^{n l} \equiv-\left\langle\dot{\mathbf{J}}_{1} G \dot{\mathbf{J}}_{1}\right\rangle \cdot \partial_{\mathbf{J}} f_{0}-\left\langle\dot{\mathbf{J}}_{1} G \dot{\mathbf{z}}_{1} \cdot \partial_{\mathbf{z}} \delta f\right\rangle,
$$

where we have used $\partial_{\theta} f_{0}=0$, i.e., that the equilibrium distribution $f_{0}$ is a function of the constants of motion only. The first contribution $\Gamma^{g l}$ is the quasilinear portion of the flux, arising from the linear term $\dot{\mathbf{z}}_{1} \cdot \partial_{\mathrm{z}} f_{0}$ in Eq.(A1), and commonly used in estimating expected transport levels. As noted in Sec. III, however, one sees that there is an additional contribution $\Gamma^{n l}$, arising from the nonlinear term in (A1), playing a role in the turbulent flux analogous to the dynamic friction in collisional transport, ${ }^{34,35}$ whose 
presence is essential in maintaining the appropriate conservation properties, and whose size in steady state may be comparable with $\Gamma^{q l}$.

Dropping the nonlinear term in Eq.(A1), one may derive ${ }^{33}$ the actionangle expression for the linear response $f^{(1)}$, and through it, for the response functions $g_{k}=-\left(n_{k} / n_{0}\right) /\left(e \phi_{k} / T\right)$ and $q_{k}=-\left(p_{k} / p_{0}\right) /\left(e \phi_{k} / T\right)$ appearing in Eq.(7) (species label $s$ suppressed). This is facilitated by writing phase functions $A(\mathbf{z})$ in the Fourier representation $A(\boldsymbol{\theta}, \mathbf{J})=\sum_{\mathbf{l}} A(\mathbf{l}, \mathbf{J}) \exp (i \mathbf{l} \cdot \boldsymbol{\theta})$, with vector Fourier harmonic index $1 \equiv\left(l_{g}, l_{b}, l_{\zeta}\right)$. In this representation one has $\dot{\mathbf{z}}_{1} \cdot \partial_{\mathbf{z}} f_{0}=-i h(\mathbf{l}, \mathbf{J}) \mathbf{l} \cdot \partial_{\mathbf{J}} f_{0}$, and $G^{-1}=\partial_{t}+i \mathbf{l} \cdot \Omega$, which is easily inverted. The full result ${ }^{33}$ is given by a sum over all 1 and modes $a$. Axisymmetry requires $l_{\zeta}=\bar{l}_{\zeta} \equiv n$, and one may take $l_{g}=\bar{l}_{g} \equiv 0$ for the low-frequency modes $\left.\omega / \Omega_{g} \ll 1\right)$ of interest here. Then one has $1 \cdot \partial_{\mathrm{J}} f_{0} \simeq f_{0}\left(\omega_{*}^{f}-1 \cdot \Omega\right) / T$, and $1 \cdot \Omega \simeq \sigma k_{\|} \overline{v_{\|}}+l \Omega_{b}+\omega_{d}$, with $\overline{v_{\|}}$the bounce/transit averaged parallel velocity, $\omega_{d} \equiv n \Omega_{\zeta}$ the toroidal drift frequency, $l \equiv l_{b}-\sigma m$, with trapping state index $\sigma=0$ for trapped particles, and $\sigma=1$ for passing particles. The linear response functions may thus be written ${ }^{35,36}$

$$
\left[\begin{array}{l}
g_{k} \\
q_{k}
\end{array}\right]=\left\langle\left[\begin{array}{c}
1 \\
u^{2} / 2
\end{array}\right]\left\{1-\sum_{l} J_{0}^{2}\left(z_{g}\right) G_{l}^{2}\left(z_{b}\right) \frac{\omega-\omega_{*}^{f}}{\omega-\sigma k_{\|} \overline{v_{\|}}-l \Omega_{b}-\omega_{d}}\right\}\right\rangle_{v},
$$

with $u \equiv v / v_{i}, \omega_{*}^{f}$ as defined in Sec. III, and $\langle\ldots\rangle_{v} \equiv \int d \mathbf{v}\left(f_{0} / n_{0}\right) \ldots$ the velocity space average over $f_{0} . J_{0}^{2}\left(z_{g}\right) G_{l}^{2}\left(z_{b}\right)$ measures the strength of the mode-particle coupling. The factor $J_{0}^{2}$ accounts for gyro-averaging, $z_{g} \equiv$ $k_{\perp} \rho_{g}$, and the factor $G_{l}^{2}\left(z_{b}\right)$ plays the analogous role for bounce/transitaveraging, reducing to $J_{l}^{2}\left(z_{b}\right)$ for a locally-eikonal mode structure $\phi_{a}(\mathbf{x})=$ $\bar{\phi}_{a}(r) \exp i\left[\int^{r} d r^{\prime} k_{r}\left(r^{\prime}\right)+m \theta+n \zeta\right]$, and becoming small for $|l| \gtrsim z_{b}$. Here, for modes with $k_{\|} \ll k_{\perp}, z_{b} \simeq k_{\perp} \rho_{b}$, with $\rho_{b}$ the particle radial drift-orbit excursion.

For simplicity we adopt the usual ordering $\omega / \Omega_{b} \gg 1$ for ions in the ITG mode. Then as discussed in Ref. 36, over the range $\Delta l \sim z_{b}$ over which $G_{l}^{2}$ is appreciable the denominator in (A4) does not change greatly, the summation over $l$ in (A4) can be approximately performed, and one may take $l_{b}=\bar{l}_{b} \equiv \sigma m$. This results in

$$
\left[\begin{array}{l}
g_{k} \\
q_{k}
\end{array}\right] \simeq\left\langle\left[\begin{array}{c}
1 \\
u^{2} / 2
\end{array}\right]\left\{1-J_{0}^{2}\left(z_{g}\right) \frac{\omega-\omega_{*}^{f}}{\omega-\sigma k_{\|} \bar{v}_{\|}-\omega_{d}}\right\}\right\rangle_{v}
$$

similar to previous expressions, ${ }^{37,38}$ including both the slablike and toroidal branches of the ITG mode. For the evaluation of Eq.(7) used in Fig. 8c, 
we expand the resonant denominator, $\left(\omega-\sigma k_{\|} \bar{v}_{\|}-\omega_{d}\right)^{-1} \simeq \omega^{-1}\left(1+\omega_{d} / \omega+\right.$ $\left.\sigma \xi^{-1} u_{\|}+\sigma \xi^{-2} u_{\|}^{2}\right)$, where $u_{\|} \equiv \bar{v}_{\|} / v_{i}$ and $\xi \equiv \omega /\left|k_{\|} v_{i}\right|$. This yields a dispersion equation

$$
0=g_{e}+\tau g_{i k}(\omega)
$$

which is cubic in $\omega$. Solving this for $\omega$ and substituting into the similar expression for $q_{i k}$ yields the proportionality factor between $Q_{k}^{q l}$ and $\left|\phi_{k}\right|^{2}$ in Fig. 8c.

Finally, we proceed to the nonlinear theory embodied in Eq.(8). In the approximation $\mathbf{l} \simeq \overline{\mathbf{l}} \equiv(0, \sigma m, n), \mathbf{l}$ is given by $\mathbf{k}$. As usual dropping the velocity-space nonlinearity the $v_{\|_{1}} \partial_{v_{\|}} \delta f$ in Eq.(4) or (A1) as down by $k_{\|} / k_{\perp}$ from the spatial nonlinearity, one can write

$$
G_{k}^{-1} f_{k}+\Delta_{k} \phi_{k}=\sum_{0=k+p+q} w_{p q} J_{o p} \phi_{p}^{*} f_{q}^{*}
$$

where $G_{k}^{-1} \equiv i(\overline{\mathbf{l}} \cdot \Omega-\omega)=i\left(\sigma k_{\|} \bar{v}_{\|}+\omega_{d}-\omega\right), \Delta_{k} \equiv J_{0 k} i\left(\overline{\mathbf{l}} \cdot \Omega-\omega_{*}^{f}\right) e f_{0} / T$, $J_{0 k} \equiv J_{0}\left(k_{\perp} \rho_{g}\right)$, and $w_{p q} \equiv(c / B) \hat{\mathbf{b}} \cdot \mathbf{p} \times \mathbf{q}$. We solve this for $f_{k}$ in the weak turbulence framework ${ }^{20}$ by iterating in powers of $\phi_{k}$. At order $\mathcal{O}(\phi)$, one has

$$
G_{k}^{-1} f_{k}^{(1)}+\Delta_{k} \phi_{k}=0
$$

giving the linear solution $f_{k}^{(1)}=-G_{k} \Delta_{k} \phi_{k}$. We introduce the field operator $\hat{\Phi}_{k}$, giving $\phi$ from $f: \hat{\Phi}_{k} f \equiv \sum_{s}\left(4 \pi e_{s} / k^{2}\right) \int d v J_{0 k} f$. Then operating on Eq.(A7) with $\hat{\Phi}_{k} G_{k}$, one finds $\epsilon^{(1)}(k) \phi_{k}=0$, where $\epsilon^{(1)} \equiv\left(1+\hat{\Phi}_{k} G_{k} \Delta_{k}\right)=$ $1+\sum_{s} g_{s k}(\omega) /\left(k \lambda_{s}\right)^{2}$ is the linear dielectric, recovering expression (A5) for $g_{s k}$.

At $\mathcal{O}\left(\phi^{2}\right),(\mathrm{A} 6)$ gives

$$
G_{k}^{-1} f_{k}^{(2)}+\Delta_{k} \phi_{k}=\sum_{0=k+p+q} w_{p q} J_{o p} \phi_{p}^{*} f_{q}^{(1) *}
$$

Symmetrizing this expression between $p$ and $q$, substituting the expression for $f_{k}^{(1)}$ just found, and again applying $\hat{\Phi}_{k} G_{k}$ yields

$$
\epsilon^{(1)}(k) \phi_{k}=-\sum_{0=k+p+q} \epsilon^{(2)}(k,-p,-q) \phi_{p}^{*} \phi_{q}^{*}
$$

with $\epsilon^{(2)}(k, p, q) \equiv \frac{1}{2} \hat{\Phi}_{k} G_{k} w_{p q}\left[J_{0 p} G_{q} \Delta_{q}-J_{0 q} G_{p} \Delta_{p}\right]$. Now writing $\epsilon^{(1)}=$ $\epsilon^{\prime}+\epsilon^{\prime \prime}$, with $\epsilon^{\prime}$ and $\epsilon^{\prime \prime}$ the reactive and dissipative parts of $\epsilon^{(1)}$, respectively, we as usual transform Eq.(A9) to the time domain, perturbatively treating $\epsilon^{\prime \prime}$ 
and the slow time deviation of $\phi_{k}$ from the real-frequency form $\exp \left(-i \omega_{r k} t\right)$. This yields $\gamma_{k}=-\left.\left(\epsilon^{\prime \prime} / \partial_{\omega} \epsilon^{\prime}\right)\right|_{\omega_{r k}}$, and Eq.(8), with

$$
M_{k p q}=i \epsilon^{(2)}(k,-p,-q) / \partial_{\omega} \epsilon^{(1)}(k) \text {. }
$$

In the fluid limit for ions, one has $J_{0} \rightarrow 1$ and $\omega \gg \overline{1} \cdot \Omega$, so that $G_{k}$ loses its velocity-space dependence. This implies that $\partial_{\omega} K_{k} \equiv \partial_{\omega}\left(\hat{\Phi}_{k} G_{k} \Delta_{k}\right) \simeq$ $-\omega^{-1} K_{k}$ in $\epsilon^{(1)}$, and that $G_{k}$ may be commuted outside the $\hat{\Phi}_{k}$ in $\epsilon^{(2)}$. Using these along with $\hat{\Phi}_{k}=\left(p^{2} / k^{2}\right) \hat{\Phi}_{p}$ and $k^{2} \lambda_{i}^{2} K_{k}=g_{k} \equiv 1+\chi_{k}$ in Eq.(A10) results in

$$
M_{k p q} \simeq w_{p q}\left(\chi_{q}^{*}-\chi_{p}^{*}\right) /\left(1+\chi_{k}\right)
$$

recovering the $M_{k p q}$ of the Terry-Horton equation. ${ }^{8}$

\section{Appendix B. The Method of Ritz, Powers, and Bengston}

We briefly synopsize the method of Ritz, et al. used in Sec. IV, referring the reader to Ref. 21 for more detail. From Eq.(8), we write the corresponding finite-difference form

$$
\phi_{k}^{\prime}=K_{k} \phi_{k}+\frac{1}{2} \sum_{0=k+p+q} Q_{k p q} \phi_{p}^{*} \phi_{q}^{*}
$$

where $\left\{K_{k}, Q_{k p q}\right\}$ are the linear and nonlinear transfer functions, closely related to $\left\{L_{k}, M_{k p q}\right\}$ (see below), $\phi_{k} \equiv \phi_{k}(t), \phi_{k}^{\prime} \equiv \phi_{k}\left(t+\tau_{1}\right)$, and $\tau_{1}$ is a time short compared with the decorrelation time $\tau_{c}$. Applying $\left\langle. . \phi_{k}^{*}\right\rangle$ and $\left\langle. . \phi_{p^{\prime}} \phi_{q^{\prime}}\right\rangle$ to (B1) yields a system of equations to solve for the $\left\{K_{k}, Q_{k p q}\right\}$ :

$$
\begin{aligned}
C_{k} & =K_{k} B_{k}+\frac{1}{2} \sum_{0=k+p+q} Q_{k p q} T_{k p q}^{*}, \\
D_{k p q} & =K_{k} T_{k p q}+Q_{k p q} E_{k p q}
\end{aligned}
$$

with $C_{k} \equiv\left\langle\phi_{k}^{\prime} \phi_{k}^{*}\right\rangle$ the (auto)correlation function, and with $D_{k p q} \equiv\left\langle\phi_{k}^{\prime} \phi_{p} \phi_{q}\right\rangle$, $E_{k p q} \equiv\left\langle\left|\phi_{p} \phi_{q}\right|^{2}\right\rangle$, and $B_{k}$ and $T_{k p q}$ defined in Sec. IV. The coefficients $B_{k}, C_{k}, D_{k p q}, E_{k p q}$, and $T_{k p q}$ are ensemble averages computed from the gk data. A quasinormal approximation due to Millionshchikov ${ }^{24}$ is used to reduce a fourth-order correlation function to the simpler form $E_{k p q}$. Solving these for $\left\{K_{k}, Q_{k p q}\right\}$, the $\left\{L_{k}, M_{k p q}\right\}$ are then given by $M_{k p q}=Q_{k p q} \exp \left(-i \Delta \theta_{k}\right) / \tau$, and $L_{k}=\left[K_{k} \exp \left(-i \Delta \theta_{k}\right)-1+i \Delta \theta_{k}\right] / \tau$, where $\exp \left(i \Delta \theta_{k}\right) \equiv C_{k} /\left|C_{k}\right|$. In Ref. 21, an iterative method was employed to solve system (B1) and (B2) 
for $\left\{K_{k}, Q_{k p q}\right\}$. In fact the system may be more simply solved. Observing that there is no summation in Eq.(B3), one may directly solve it for $Q_{k p q}$ :

$$
Q_{k p q}=\left(D_{k p q}-K_{k} T_{k p q}\right) / E_{k p q}
$$

Using this in Eq.(B2) to eliminate $Q_{k p q}$ and solving for $K_{k}$, one has

$$
K_{k}=\left(C_{k}-\frac{1}{2} \sum\left(D_{k p q} T_{k p q}^{*} / E_{k p q}\right)\right) /\left(B_{k}-\frac{1}{2} \sum\left(\left|T_{k p q}\right|^{2} / E_{k p q}\right)\right) .
$$

Substituting this back into (B4) yields the solution for $Q_{k p q}$. 


\section{References}

${ }^{1}$ A.A. Galeev, R.Z. Sagdeev, Sov.Phys-JETP 26, 233 (1969).

${ }^{2}$ P. H. Rutherford, Phys. Fluids 13, 482 (1970).

${ }^{3}$ F. L. Hinton and R. D. Hazeltine, Rev. Mod. Phys 48,Part 1, 239 (1976).

${ }^{4}$ P. C. Liewer, Nucl. Fusion 25, 543 (1985).

${ }^{5}$ W.W. Lee, Physics of Fluids 26, 556 (1983).

${ }^{6}$ S.E. Parker, W.W. Lee, R.A. Santoro, Phys. Rev. Letters 71, 2042 (1993).

${ }^{7}$ A. Hasegawa, K. Mima, Phys.Rev.Lett. 39, 205 (1977).

${ }^{8}$ P.W. Terry, W. Horton, Phys.Fluids 25, 491 (1982).

${ }^{9}$ R.E. Waltz, Phys.Fluids 26, 169 (1983).

${ }^{10} \mathrm{~W}$. Horton, Phys.Fluids 29, 1491 (1986).

${ }^{11}$ J. Bowman, J.A. Krommes, M. Ottaviani, Physics of Fluids-B 5, 3558 (1993).

${ }^{12}$ J. Bowman, J.A. Krommes, M. Ottaviani, (in draft, 1994).

${ }^{13}$ S.E. Parker, W.W. Lee, Physics of Fluids-B 5, 77 (1993).

${ }^{14}$ G. Hu, J.A. Krommes, Physics of Plasmas 1, 863 (1994).

${ }^{15}$ T.S. Hahm, Physics of Fluids31, 2670 (1988).

${ }^{16}$ G. W. Hammett, M. A. Beer, W. Dorland, S. C. Cowley, and S. A. Smith, Plasma Phys. Control. Fusion 35, 973 (1993).

${ }^{17}$ R.E. Waltz, G.D. Kerbel, and J. Milovich, General Atomics Report GAA21565 (to appear in Physics of Plasmas, 1994).

${ }^{18}$ B.I. Cohen, T.J. Williams, A.M. Dimits, and J.A. Byers, Phys. Fluids B 52967 (1993).

${ }^{19}$ M. Zarnstorff, et al., Proceedings of the Twelfth International Conference on Plasma Physics and Controlled Nuclear Fusion Research (Nice, France, October, 1988) (International Atomic Energy Agency, Vienna, Austria, 1989), Paper IAEA-CN-50/A-3-3. 
${ }^{20}$ R.Z. Sagdeev, A.A. Galeev, Nonlinear Plasma Theory, T.M. O'Neil and D.L. Book (eds), W.A. Benjamin, Inc. New York (1969).

${ }^{21}$ Ch.P. Ritz, E.J. Powers, R.D. Bengston, Physics of Fluids-B 1, 153 (1989).

${ }^{22}$ S. Assadi, S.C. Prager, K.L. Sidikman, Phys. Rev. Letters 69, 281 (1992).

${ }^{23}$ N. Aubrey, P. Holmes, J.L. Lumley, E. Stone, in Advances in Fluid Turbulence, Doolen, Ecke, Holm, Steinberg (eds.), North Holland, Amsterdam (1989), p.1-10.

${ }^{24}$ M.D. Millionshchikov, Dokl. Akad. Nauk SSSR 32, 611 (1941).

${ }^{25}$ F.W. Perkins, C.W. Barnes, D.W. Johnson, S.D. Scott, et al., Physics of Fluids-B 31, 477 (1993).

${ }^{26}$ S.M. Kaye, C.W. Barnes, M.G. Bell, J.C. DeBoo, et al., Phys. Fluids-B 2 2926 (1990).

${ }^{27}$ M.G. Bell, L.R. Grisham, R.V. Budny, J.D. Strachan, Bull.Am.Phys.Soc. 38, paper 2F08 (St. Louis, 1993).

${ }^{28}$ S.D. Scott, et al., "Heat and Particle Transport in Deuterium-Tritium Plasmas, in draft (1994).

${ }^{29}$ W.M. Tang, in Theory of Fusion Plasmas, edited by J. Vaclavik, F. Troyon, and E. Sindoni, Proceedings of the Joint Varenna-Lausanne International Workshop, Varenna, Italy (August, 1990) (Editrice Compositori, Bologna, 1990) pp.31-44.

${ }^{30}$ J.W. Connor, J.B. Taylor, Nucl.Fusion 17, 1047 (1977).

${ }^{31}$ R.A. Santoro, Ph.D. Dissertation, Princeton University (June, 1994).

${ }^{32}$ S.D. Scott, C.W. Barnes, L.R. Grisham, G. Hammett, W.W. Heidbrink, et al., Proceedings of the 13th International Conference on Plasma Physics and Controlled Nuclear Fusion Research (Washington, DC, October, 1990) (International Atomic Energy Agency, Vienna, Austria), Paper IAEA-CN-53/A-3-6 (1990).

${ }^{33}$ A. N. Kaufman, Phys. Fluids 15, 1063 (1972).

${ }^{34}$ T.H. Dupree, Phys.Rev.Lett. 25, 789 (1970). 
${ }^{35}$ H.E. Mynick and R.E. Duvall, Phys. Fluids-B 1, 750 (1989).

${ }^{36}$ H.E. Mynick, Phys.Fluids-B 4, 1229 (1992).

${ }^{37}$ G. Rewoldt, W.M. Tang, E.A. Frieman, Phys.Fluids 211513 (1978).

${ }^{38}$ H. Biglari, P. H. Diamond, M. N. Rosenbluth, Phys.Fluids-B 1, 109 (1989). 


\section{Figures}

FIG. 1. Geometry and parametrization of the simulation volume.

FIG. 2. Radially-averaged ion heat flux $\bar{Q}_{i}^{r}$ versus time for a typical gk simulation, showing the three characteristic phases (a-c) discussed in Sec. II.

FIG. 3. For linear growth phase-a, (a)contours of constant potential $\phi(r, \theta, \zeta)$ in poloidal projection, (b) $\phi(r, \theta, \zeta)$ in the midplane, and (c) $|\phi(r, m, n)|$ over the $(m, n)$-plane.

FIG. 4. As Fig. 3, but for nonlinearly saturated phase-c.

FIG. 5. Plots of the (a)Bohm and (b)gyroBohm expressions for the heat flux $Q$ versus minor radius, for the temperature profile used in the simulations.

FIG. 6. Time-averaged value of the numerical heat flux $\bar{Q}_{g k}^{t}$ versus minor radius.

FIG. 7. Running sum $Q_{j} \equiv \sum_{i=1}^{j} Q_{k_{i}}$ of the largest $\mathrm{j}$ contributors to the ion heat flux $Q\left(r=r_{0}\right)$ versus $\mathrm{j}$.

FIG. 8. Time-dependences of (a)the $N_{k} / 2=6$ largest contributing pairs $Q_{ \pm k}$ to the heat flux $Q$ at $r=r_{0}$, (b) $\phi_{k}(t)$ corresponding to the $Q_{k}$ in (a), and (c) $Q_{k}(t)$ computed using the $\phi_{k}$ from (b) in a quasilinear expression.

FIG. 9. Power spectrum of the $n=4$ component of $\phi$ from the simulation, observed at $r=a / 2, \theta=0$ in nonlinear phase-c.

FIG. 10. Coefficients (a) $L_{k}$ and (b) $M_{k p q}$ used to generate the test data to benchmark the implementation of the method of Ref. 21. The numbers $j_{k}=1, . . N_{K}$ in (a) are mode labels.

FIG. 11. Inferred $L_{k}$ and $M_{k p q}$ for the system with real coefficients in Fig. 10, for ensembles with $N_{r l}=(\mathrm{a}, \mathrm{b}) 2000$, (c,d) 100, (e,f) 30, and (g,h) 5 .

FIG. 12. $T_{k p q}$ over the $\mathbf{p}=\left(m_{2}, n_{2}\right)$ plane computed from the gk data for $k=(-9,4)$, a typical dominant contributor to transport. 
FIG. 13. Coefficients $(\mathrm{a}, \mathrm{b}) L_{k}$ and (c) $M_{k p q}$ computed from the gk data. The $\mathrm{k}$-value for (c) is the same as for Fig. 12. As in Fig. 10, the numbers $j_{k}$ are mode labels, here for the reduced gk system.

FIG. 14. (a)Plot of power transfer function $T(k, p, q)$ over the $\left(n, n_{2}\right)$ plane for the gk data of Figs. 12 and 13. (b)Total nonlinear power transfer $\sum_{p} T(k, p, q)$ from modes $\mathrm{p}$ into mode $\mathrm{k}=(m, n)$ versus $n$. The numbers are mode labels $j_{k}$, as in Fig. 10. (c)Power spectrum $B_{k}$ versus $n$.

FIG. 15. Parameter scan of normalized heat flux $Q$ versus $q_{a}$, for gk tokamaks with $a / \rho_{g}=64, q_{0}=1.25$.

FIG. 16. Locus of the rational surfaces $q(r)=2,3, . .8$ versus $q_{a}$, showing their intersection with the $r=r_{0}$ surface. At the right is shown $\kappa_{T}(r / a)$ providing the drive for the instabilities.

FIG. 17. Scaling of $Q$ with $a / \rho_{g}$, with $q_{0}=1.25$ and $q_{a}=4.25$.

FIG. 18. Power spectra $B_{k}$ versus $k_{r} \rho_{g}$ for simulations with $a / \rho_{g}=64$ and 128. 


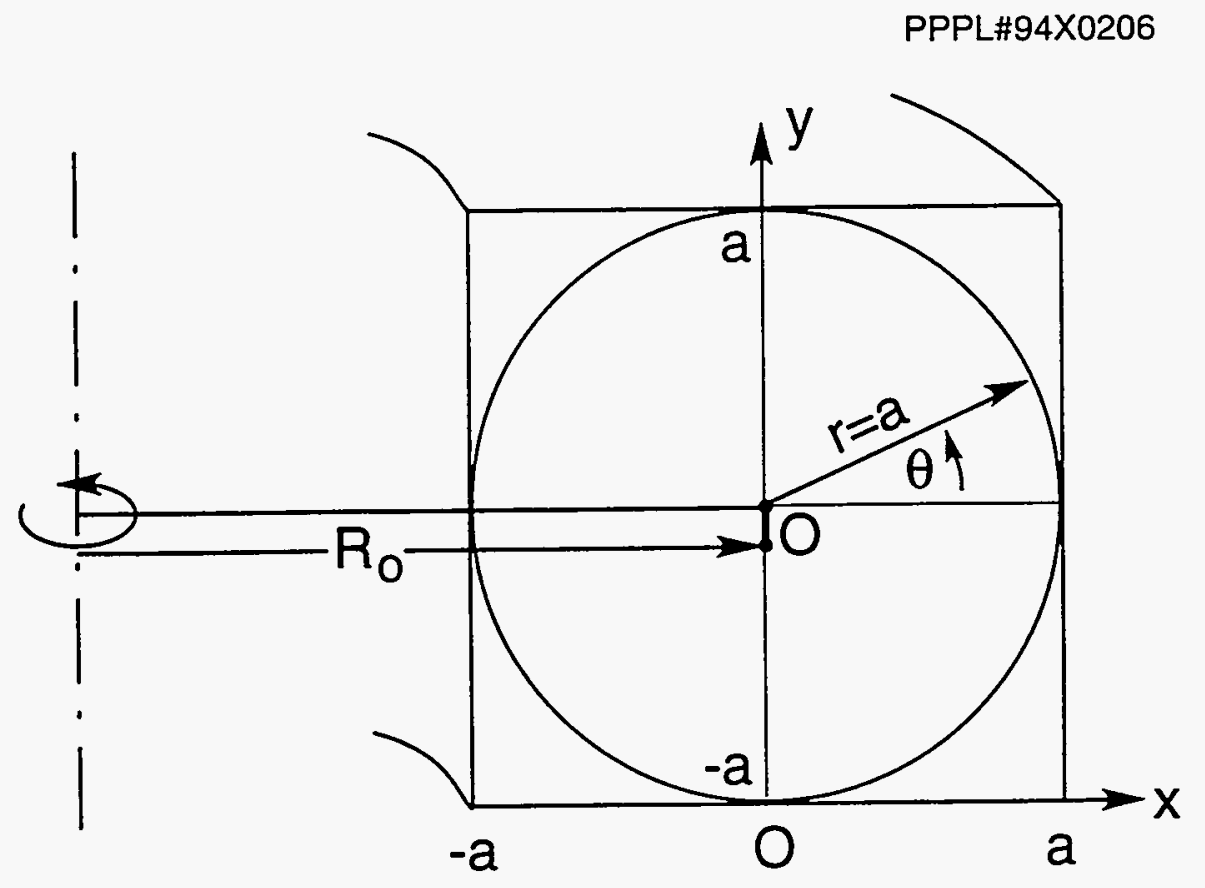

Fig. 1 
PPPL\#94X0207

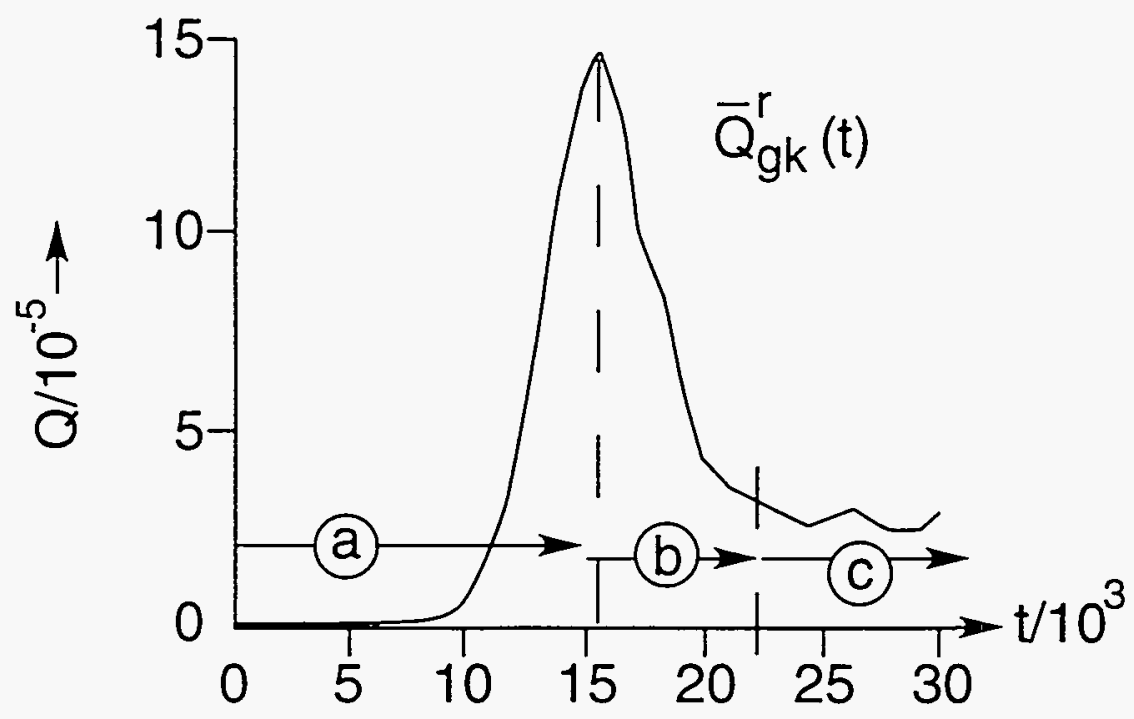

Fig. 2 
PPPL\#94X0208
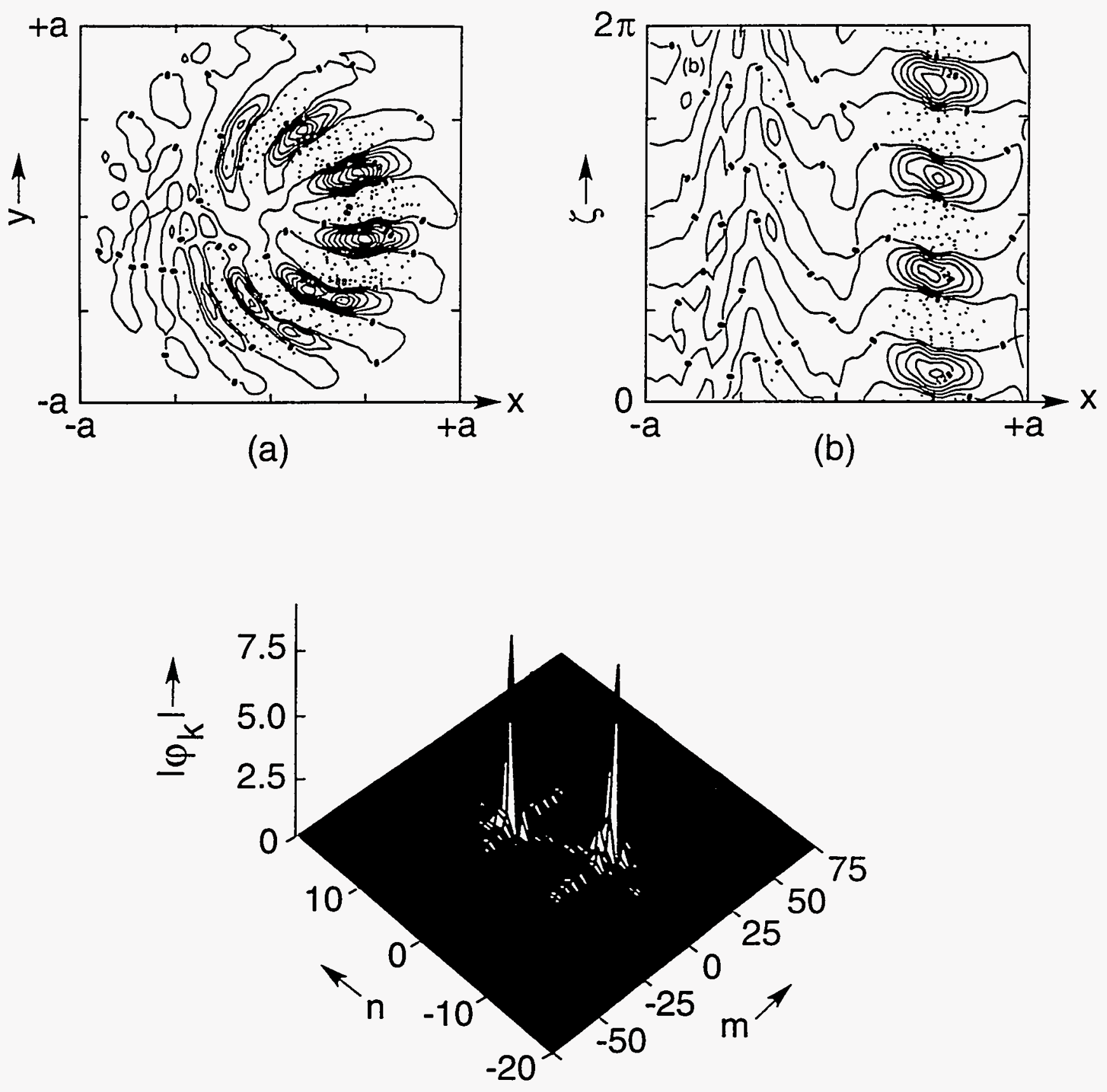

(c)

Fig. 3 
PPPL\#94X0209
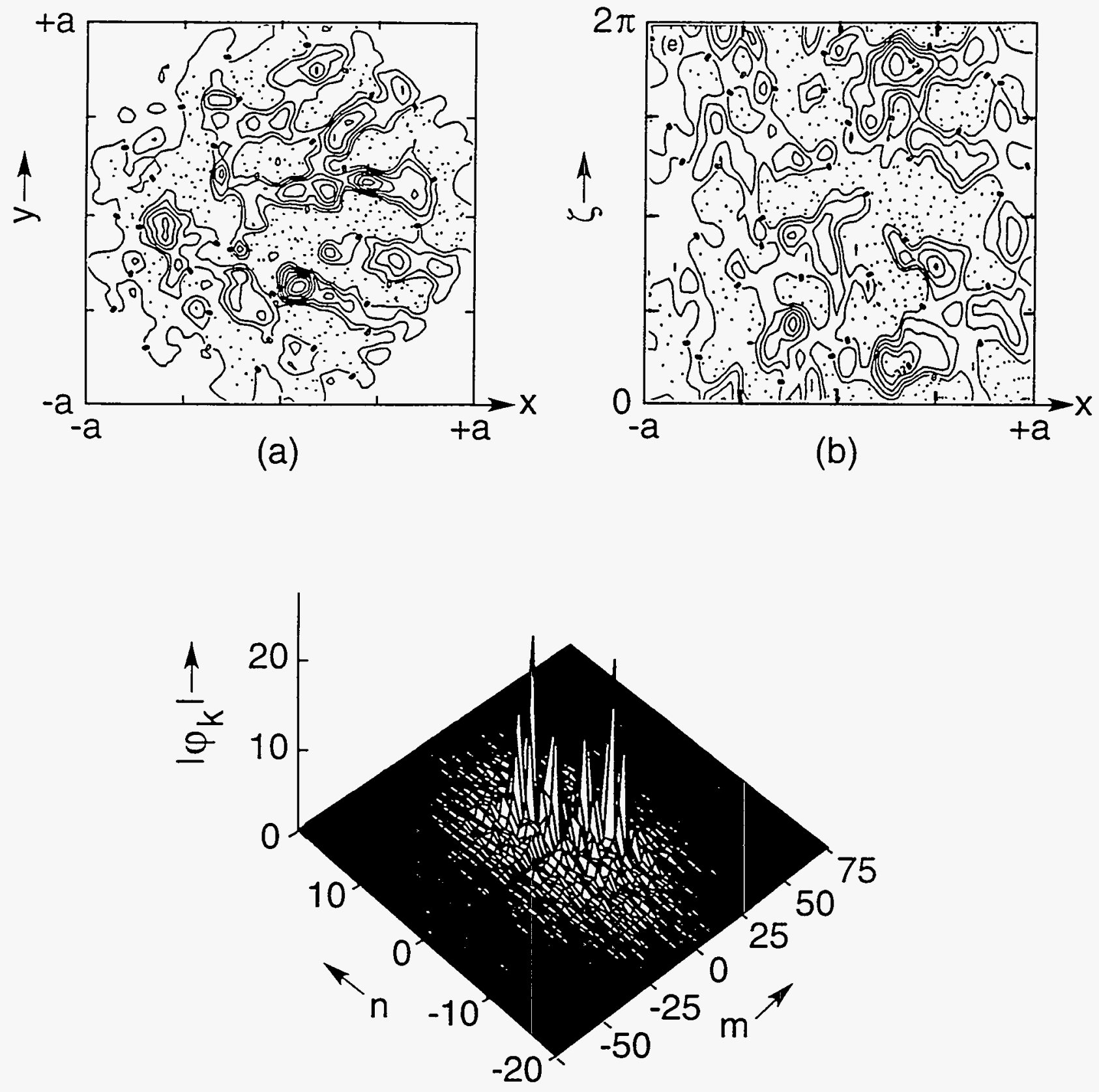

(c)

Fig. 4 

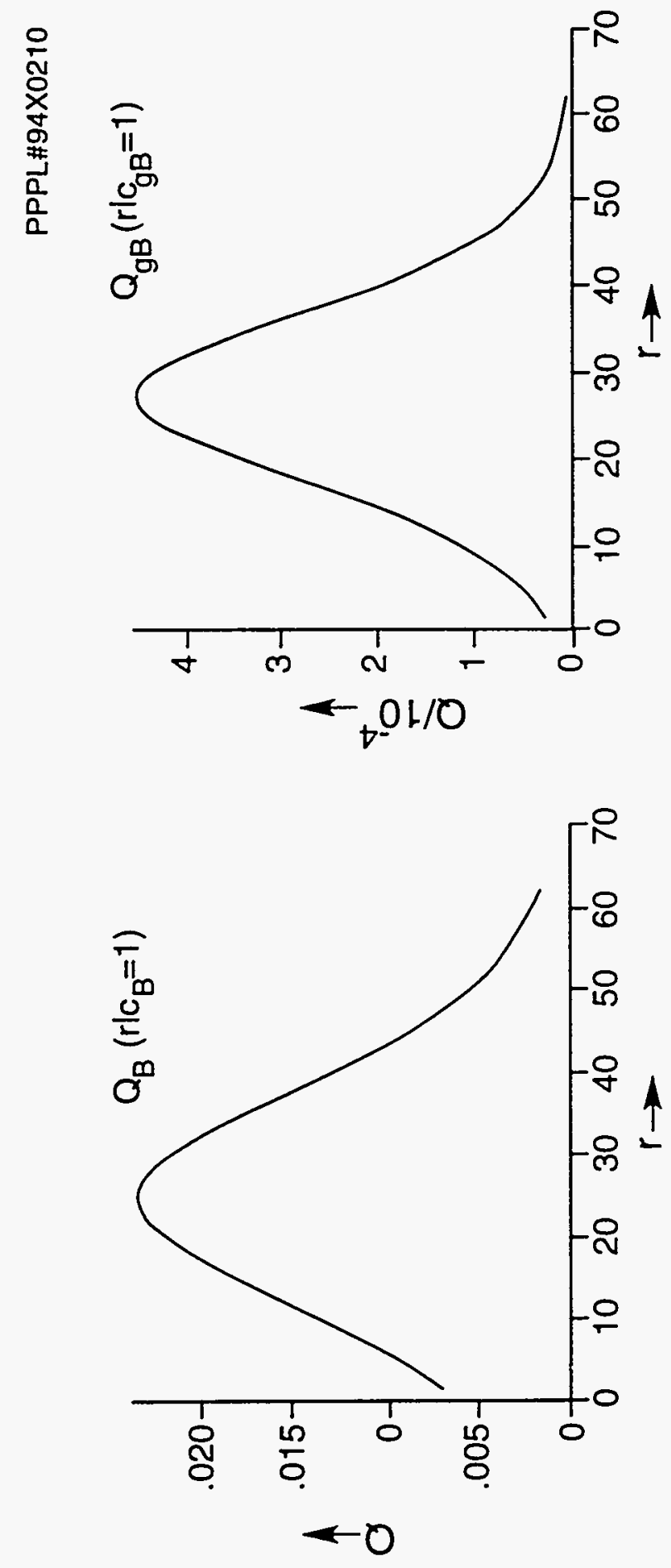

ir 


\section{PPPL\#94X0211}

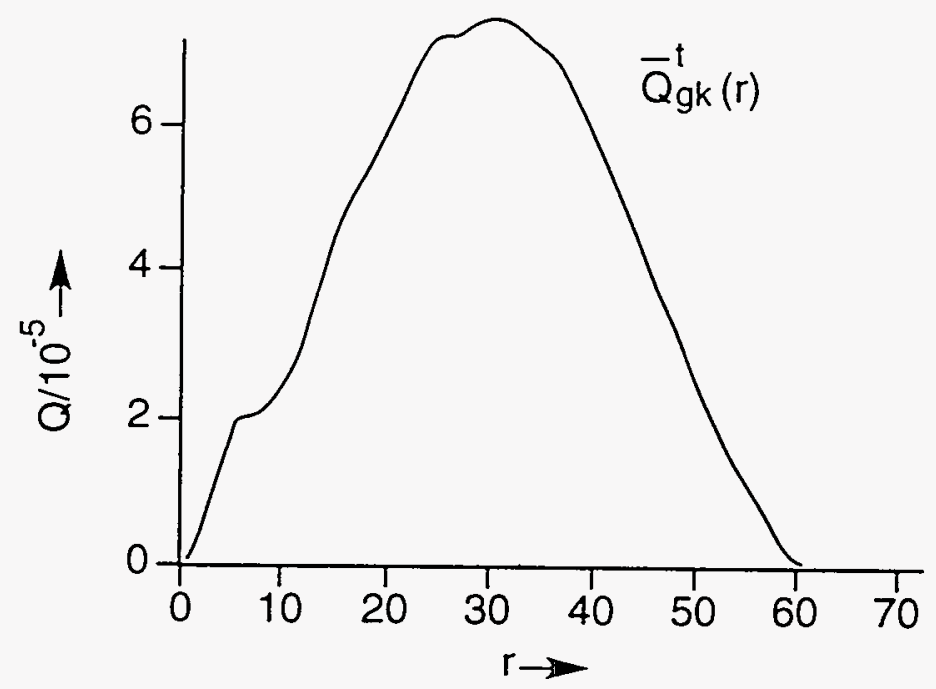

Fig. 6 


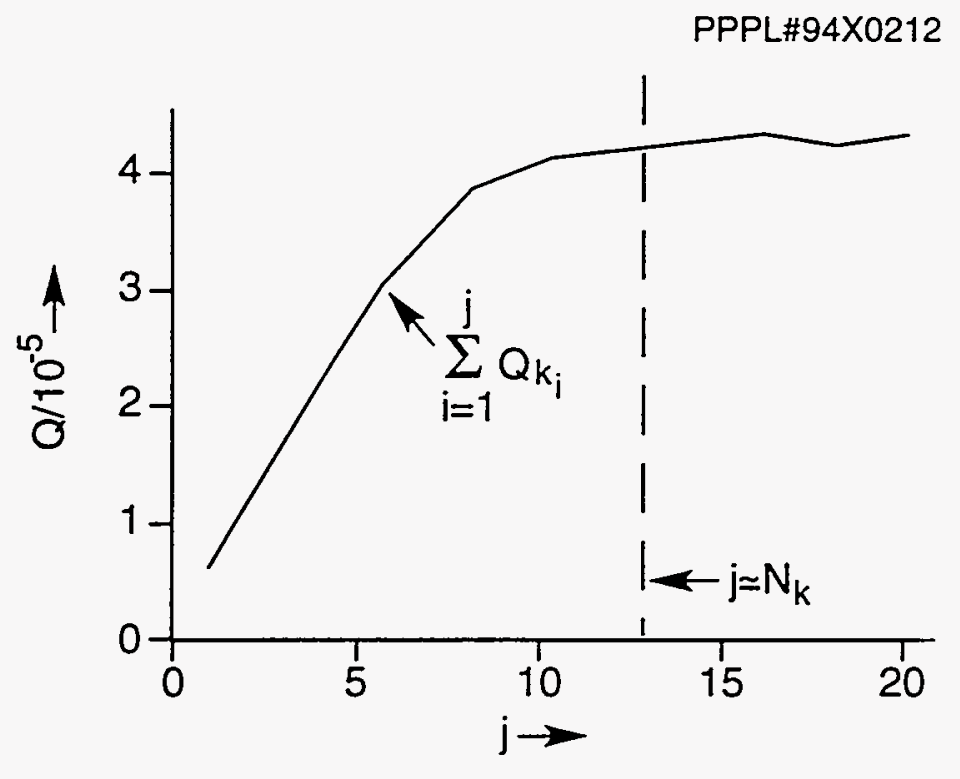

Fig. 7 


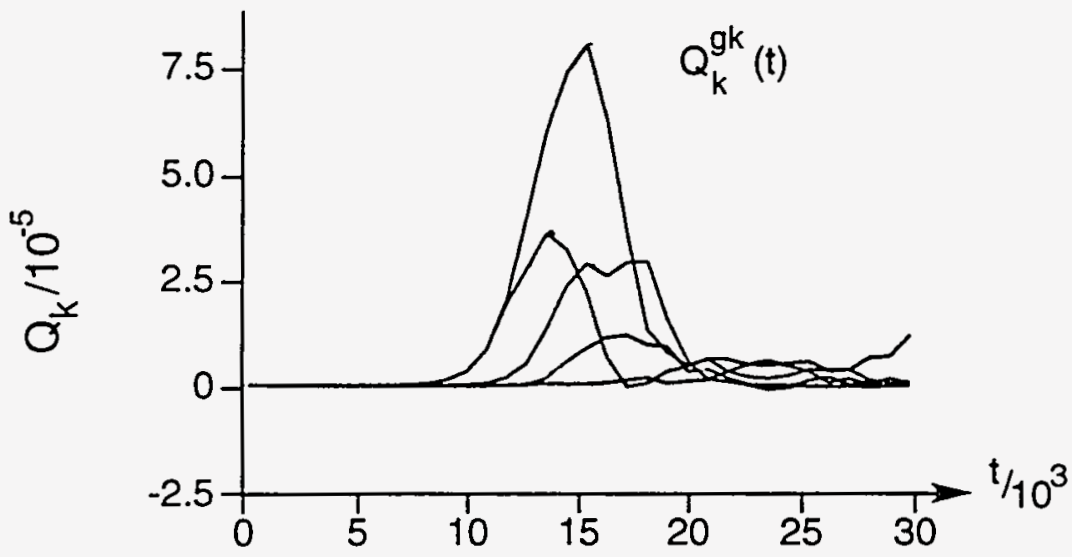

(a)

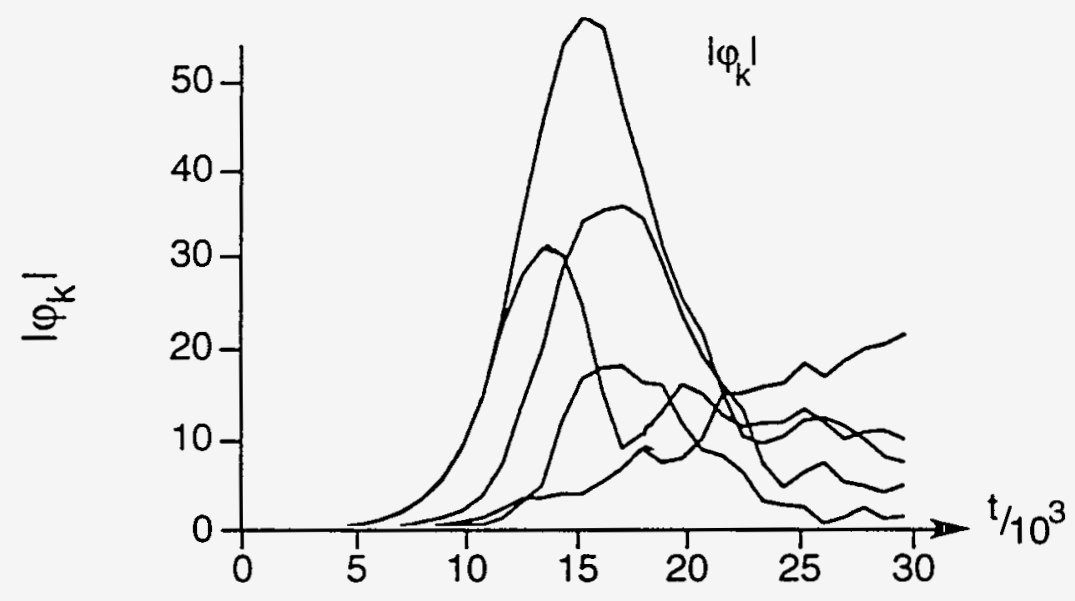

(b)

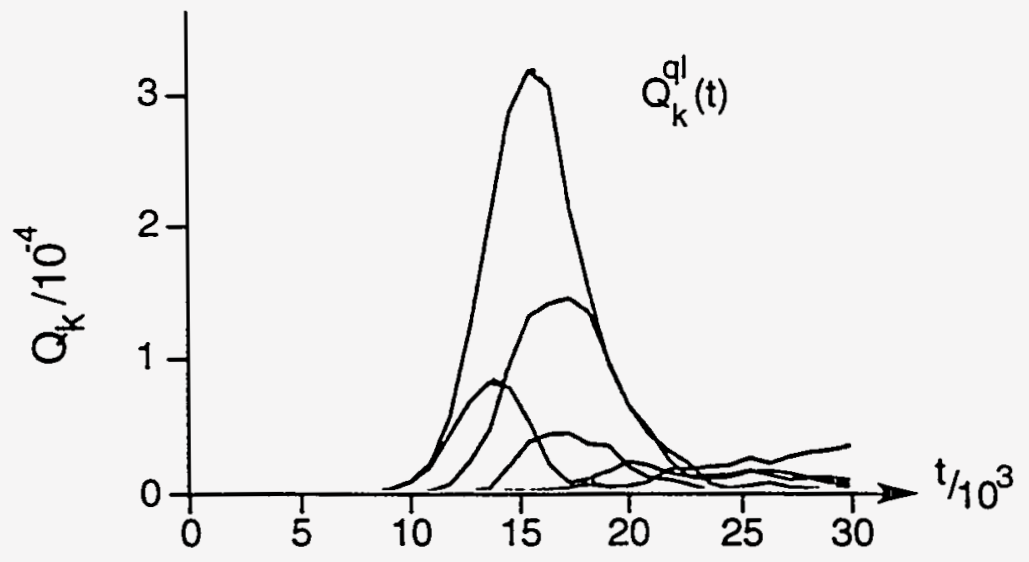

(c)

Fig. 8 


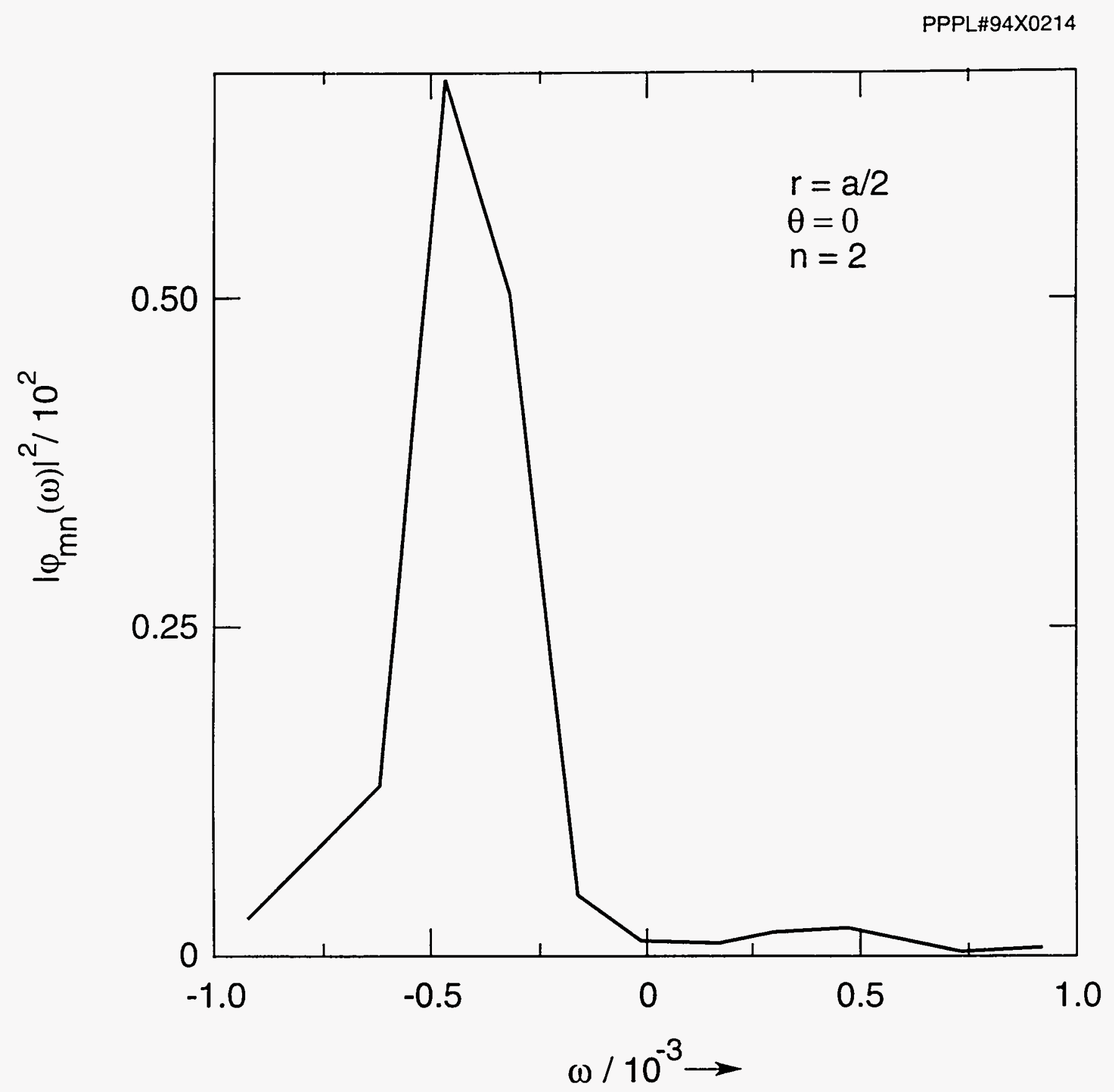

Fig. 9 

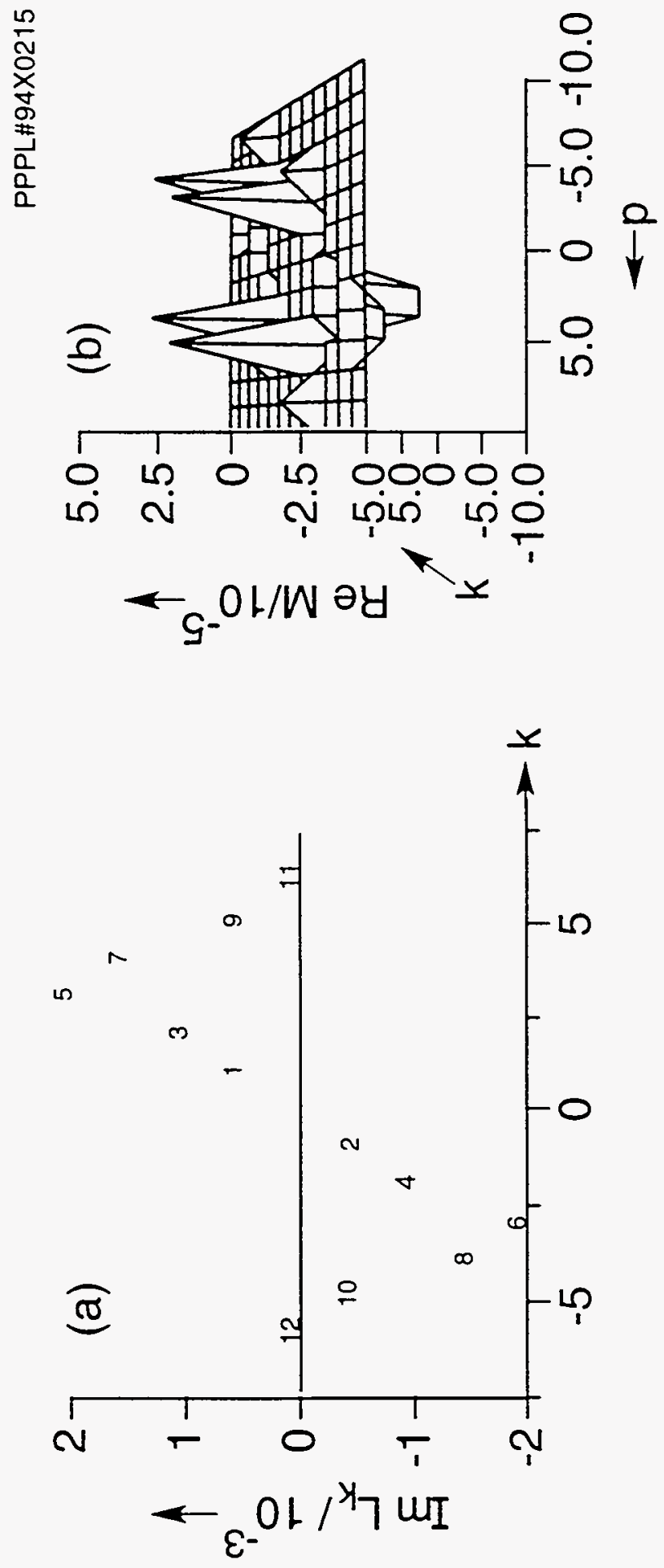

Fig. 10 

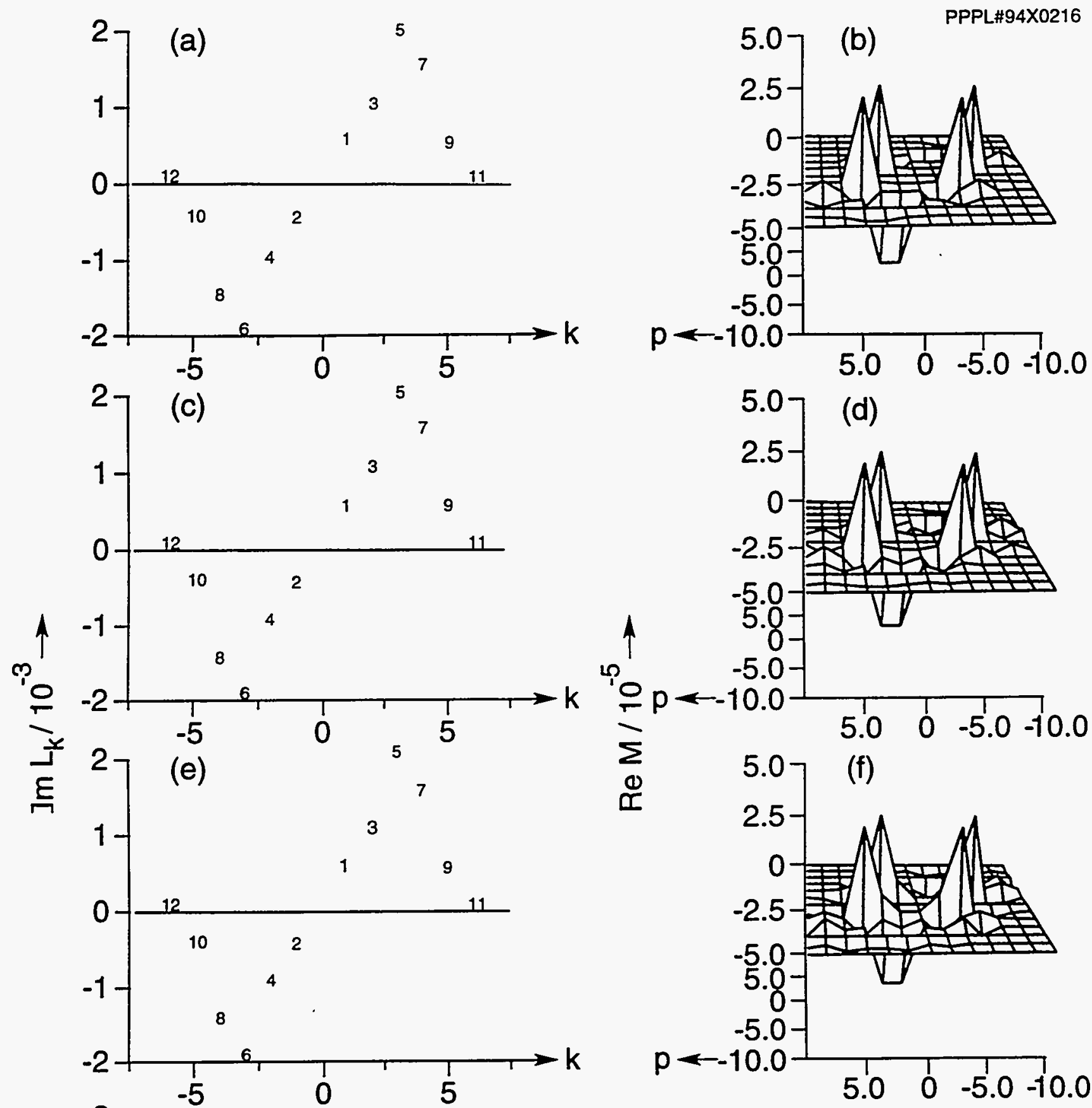

$\sum_{\substack{0 \\ \mathbb{1}}}$
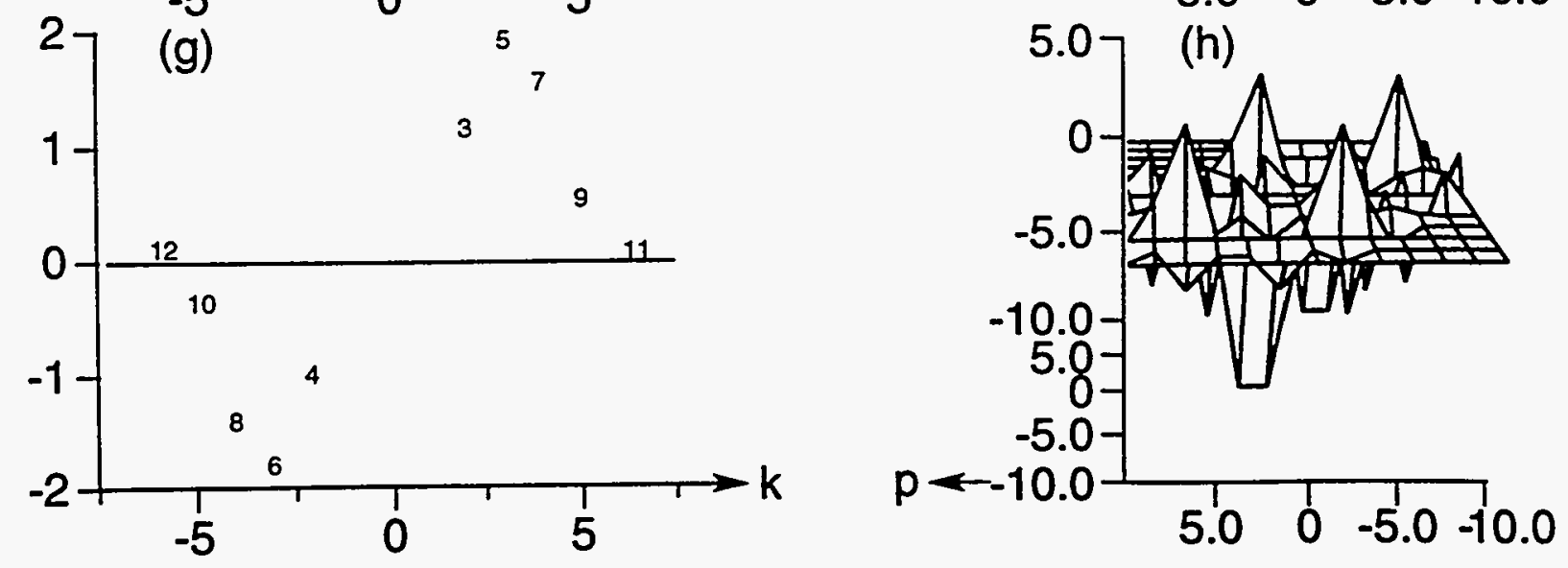

Fig. 11 
PPPL\#94X0217

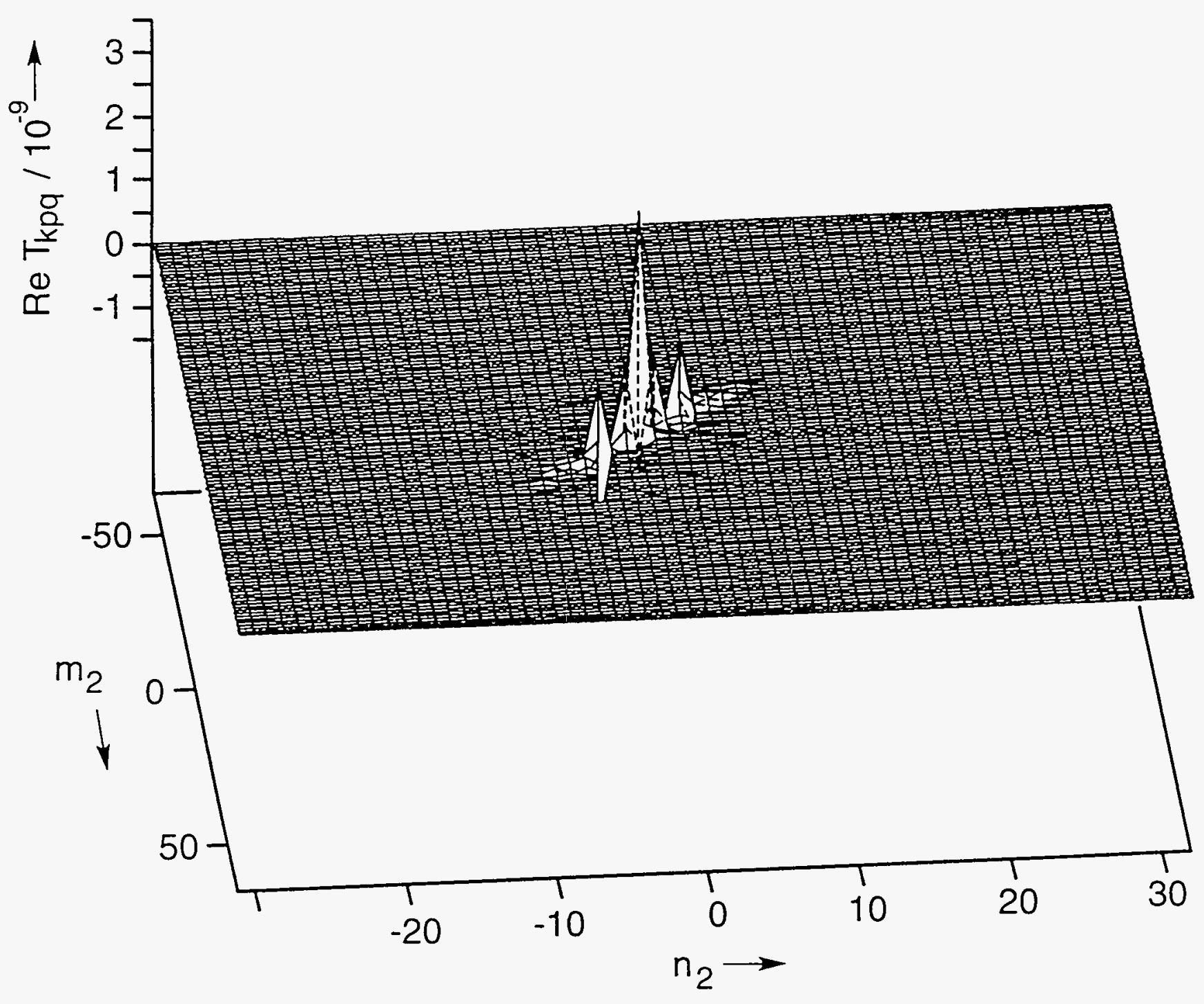

Fig. 12 

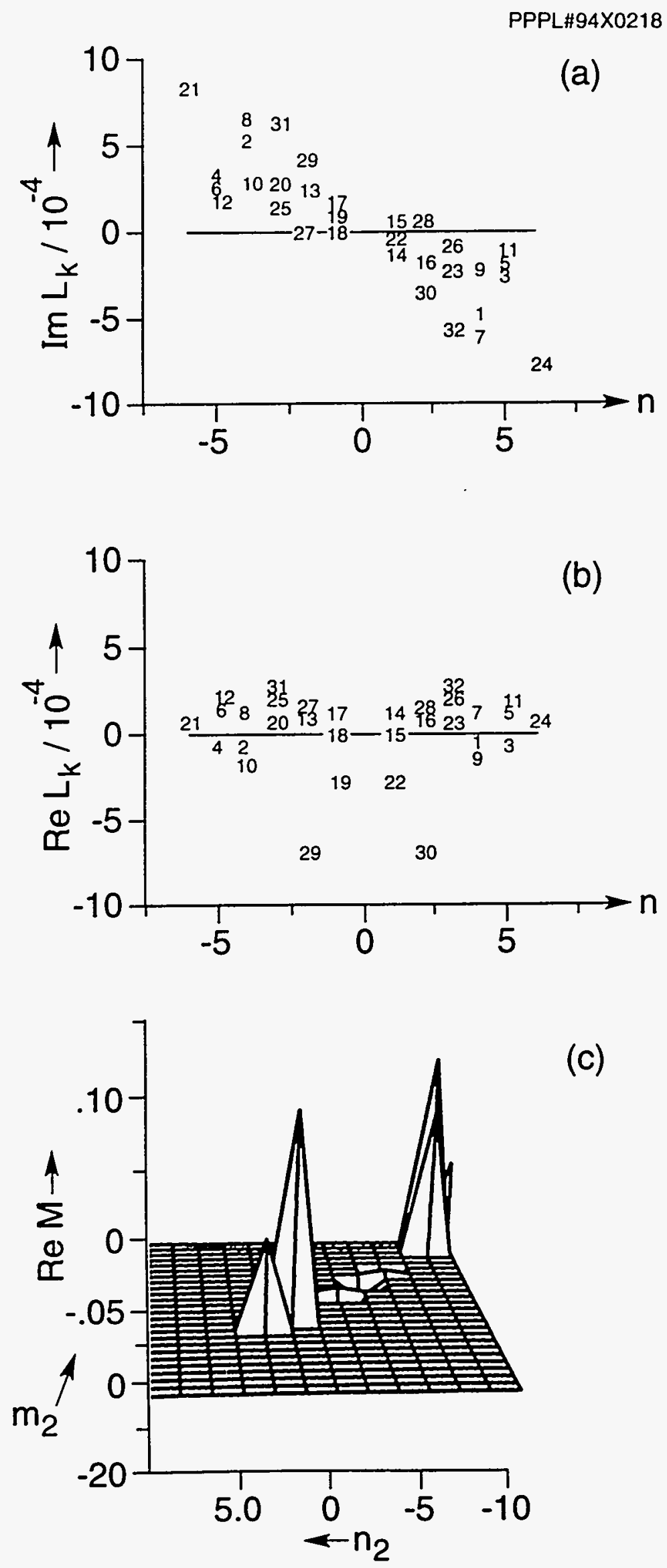

(c)

Fig. 13 


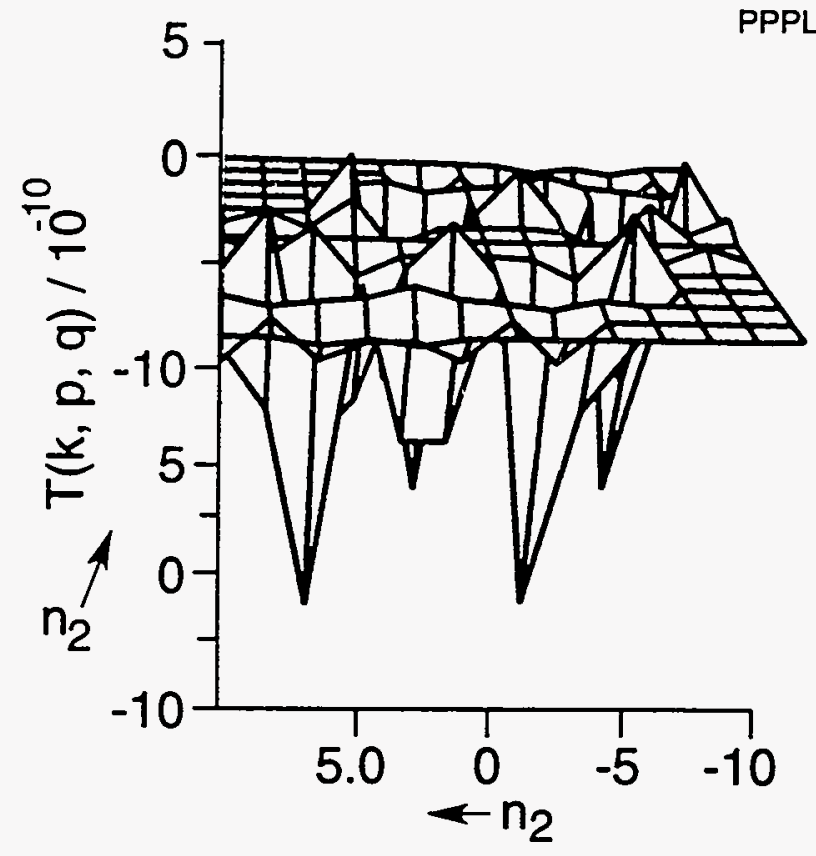

(a)
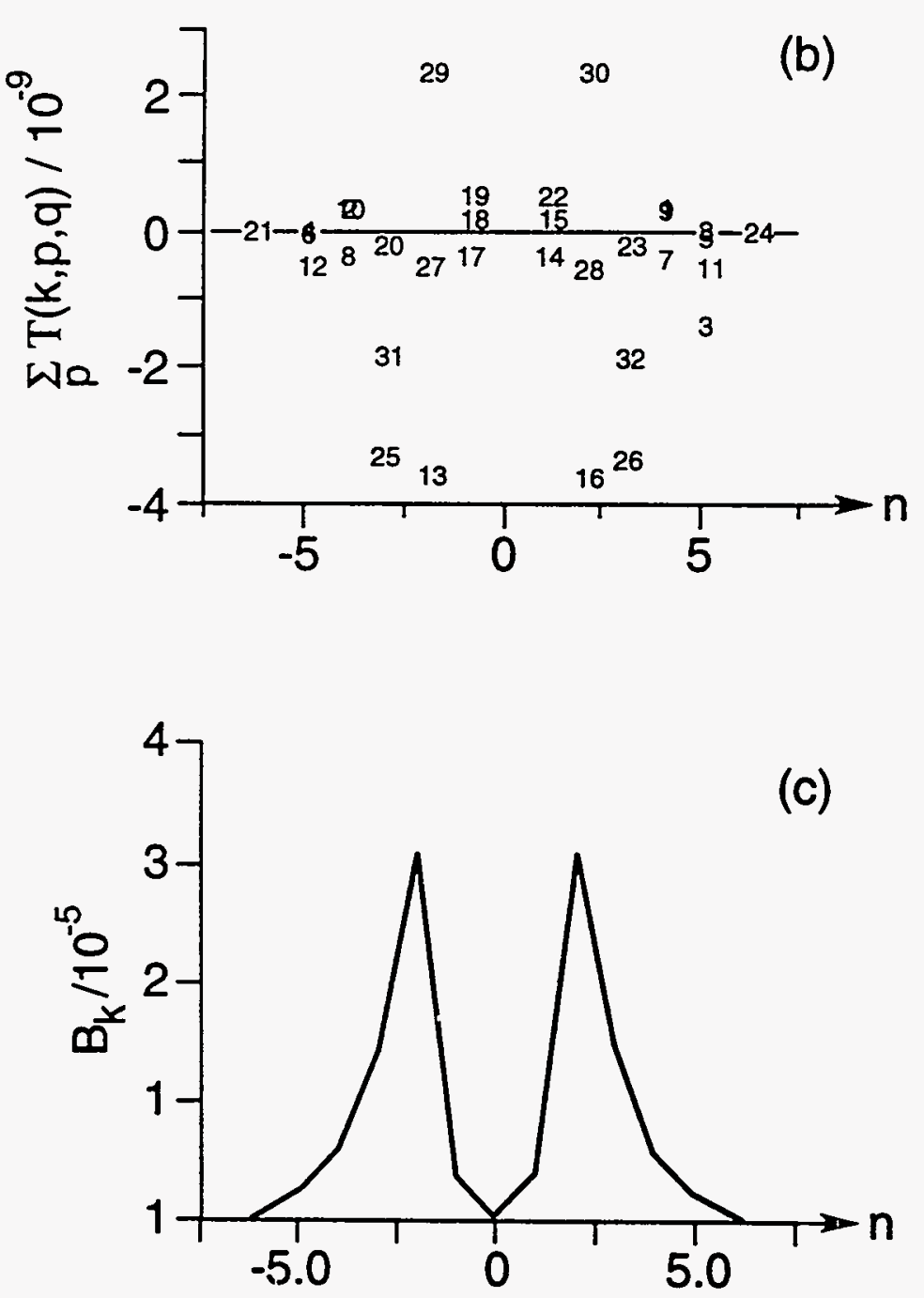

Fig. 14 


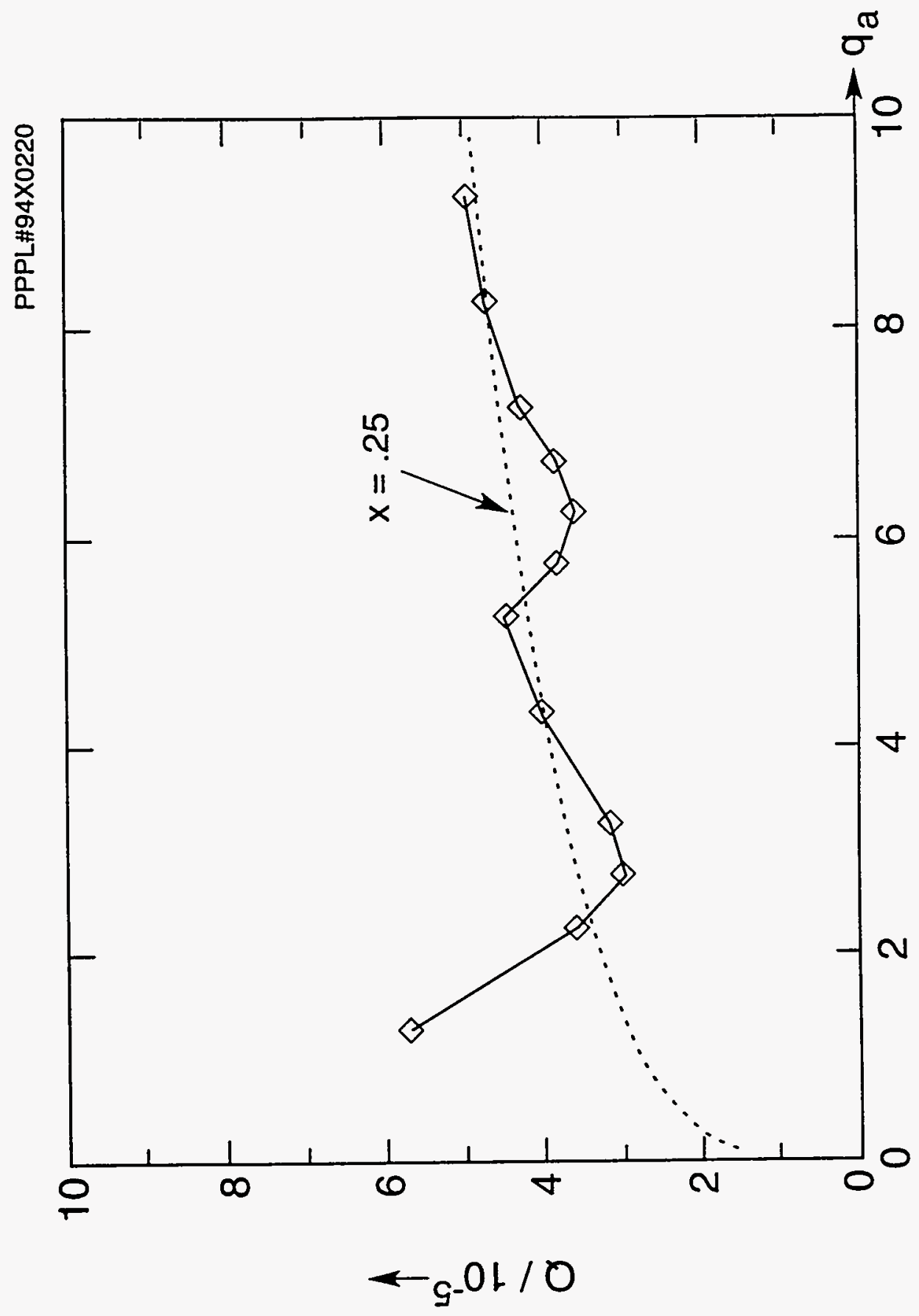

Ln
$\dot{\sigma}$
$\dot{\pi}$ 


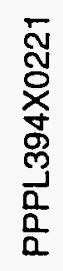
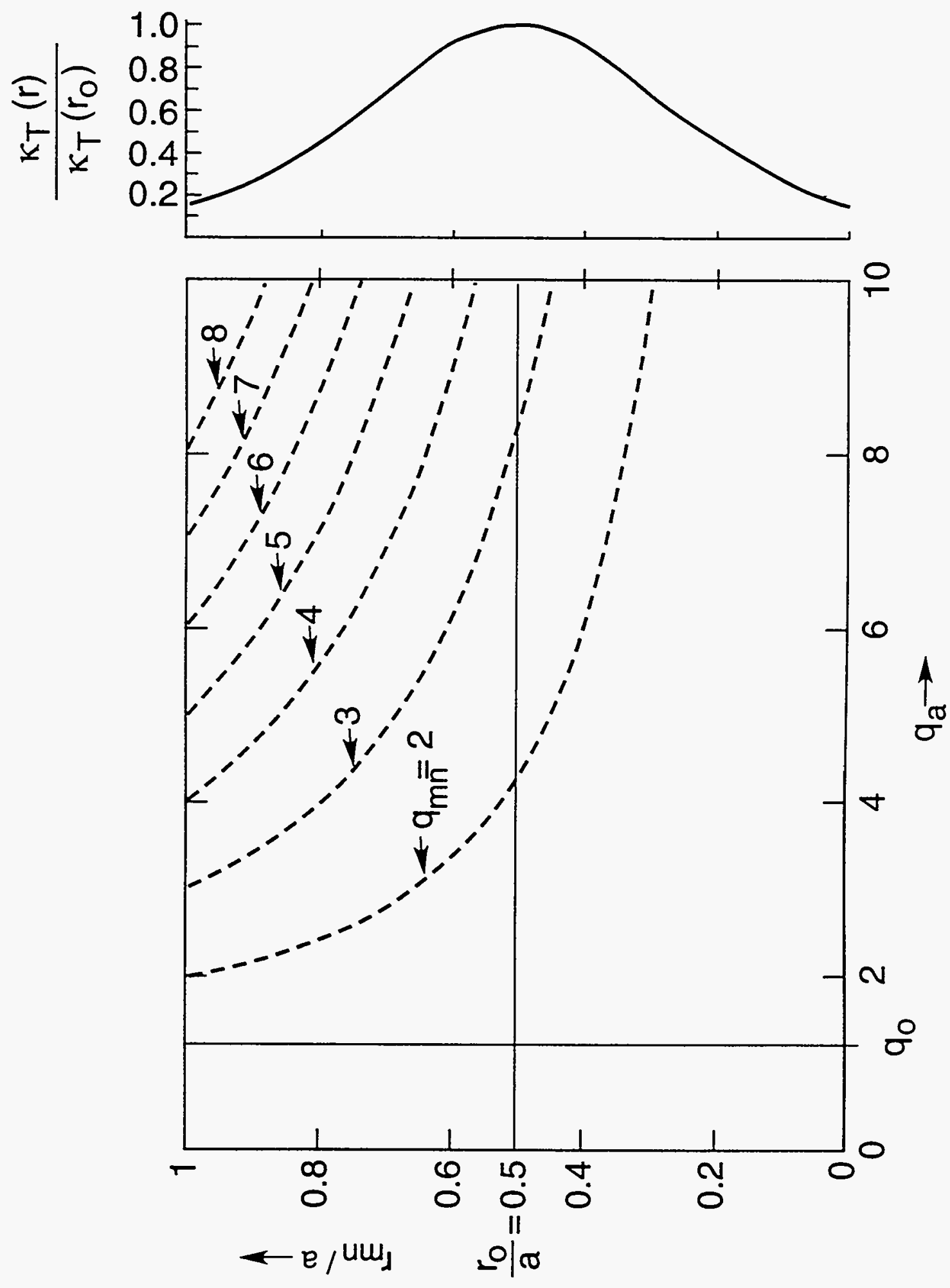


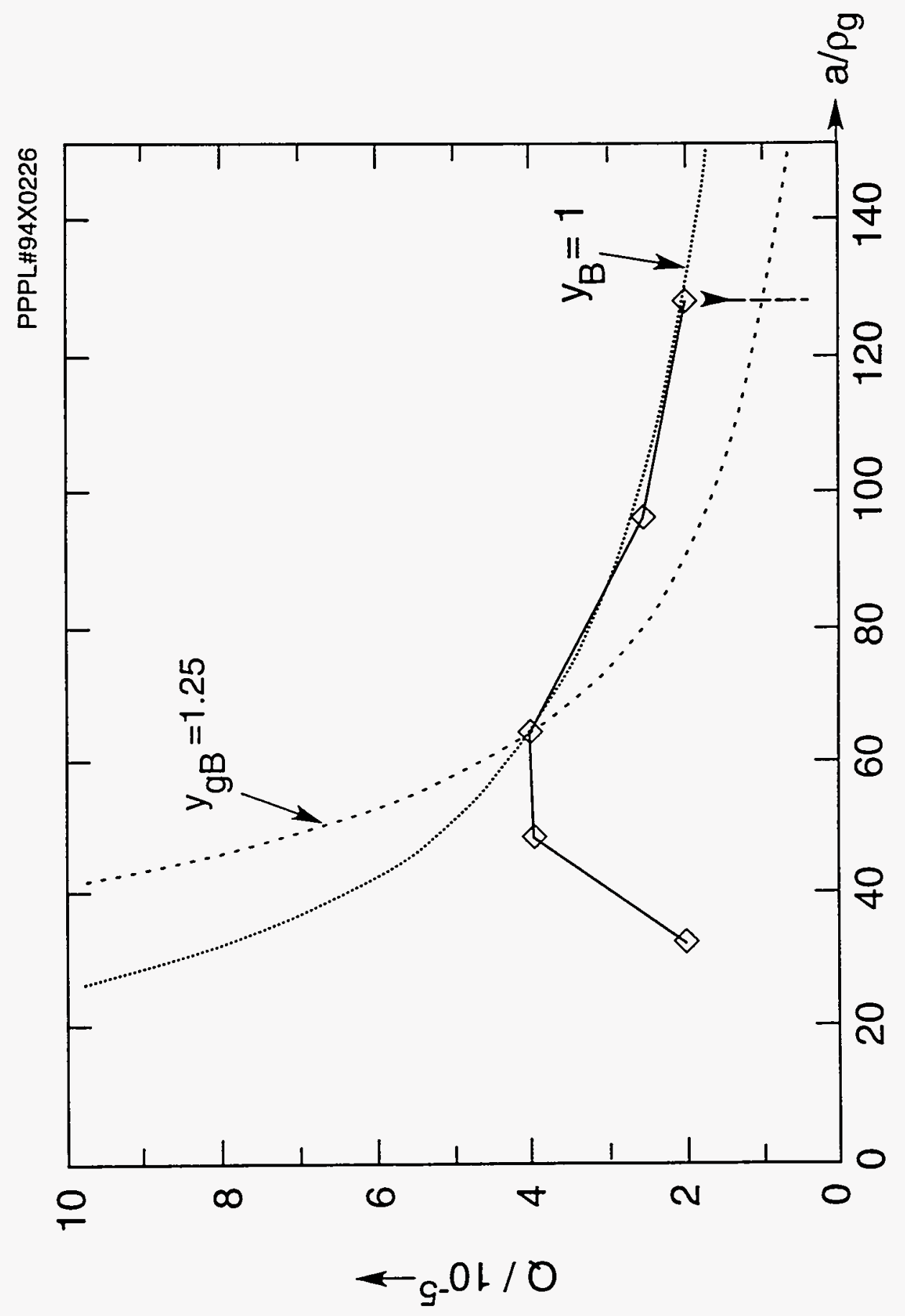




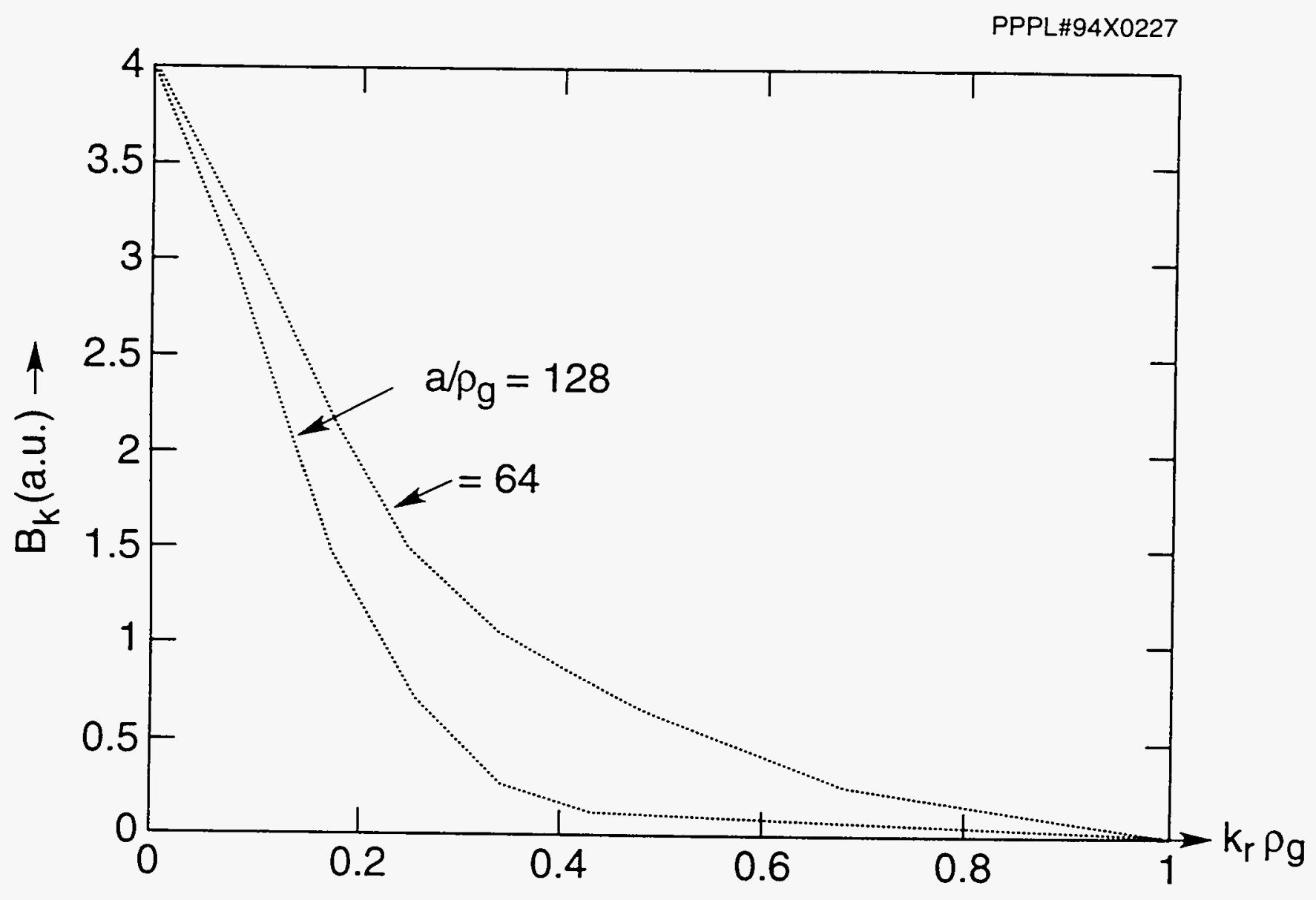

Fig. 18 
Dr. F. Paoloni, Univ. of Wollongong, AUSTRALIA

Prot. R.C. Cross, Univ. of Sydney, AUSTRALIA

Plasma Research Lab., Australian Nat. Univ., AUSTRALIA

Prof. I.R. Jones, Flinders Univ, AUSTRALIA

Prof. F. Cap, Inst. for Theoretical Physics, AUSTRIA

Prot. M. Heindler, Institut für Theoretische Physik, AUSTRIA

Prof. M. Goossens, Astronomisch Instituut, BELGIUM

Ecole Royale Militaire, Lab. de Phy. Plasmas, BELGIUM

Commission-European, DG. XII-Fusion Prog., BELGIUM

Prol. R. Bouciqué, Aijksuniversiteit Gent, BELGIUM

Dr. P.H. Sakanaka, Instituto Fisica, BRAZIL

Prof. Dr. I.C. Nasćimento, Instituto Fisica, Sao Paulo, BRAZIL Instituto Nacional De Pesquisas Espaciais-INPE, BRAZIL

Documents Office, Atomic Energy of Canada Ltd., CANADA

Ms. M. Morin, CCFMTokamak de Varennes, CANADA

Dr. M.P. Bachynski, MPB Technologies, Inc., CANADA

Dr. H.M. Skarsgard, Univ. of Saskatchewan, CANADA

Prof. J. Teichmann, Univ. of Montreal, CANADA

Prol. S.R. Sreenivasan, Univ. of Calgary, CANADA

Prof. T.W. Johnston, INRS-Energie, CANADA

Dr. R. Bolton, Centre canadien de fusion magnétique, CANADA

Dr. C.R. James,, Univ. of Alberta, CANADA

Dr. P. Lukác, Komenského Universzita, CZECHO-SLOVAKIA

The Librarian, Culham Laboratory. ENGLAND

Library, R61, Rutherford Appleton Laboratory, ENGLAND

Mrs. S.A. Hutchinson, JET Library, ENGLAND

Dr. S.C. Sharma, Univ. of South Pacific, FIJI ISLANDS

P. Măhonnen, Univ. of Helsinki, FINLAND

Prof. M.N. Bussac, Ecole Polytechnique,, FRANCE

C. Mouttet, Lab. de Physique des Milieux lonisés, FRANCE

J. Radet, CEN/CADARACHE - Bat 506, FRANCE

Prof. E. Economou, Univ. of Crete, GREECE

Ms. C. Rinni, Univ. of loannina, GREECE

Preprint Library, Hungarian Academy of Sci, HUNGARY

Dr. B. DasGupta, Saha Inst. of Nuclear Physics, INDIA

Dr. P. Kaw, Inst. for Plasma Research, INDIA

Dr. P. Rosenau, Israel Inst. of Technology, ISRAEL

Librarian, Intemational Center for Theo Physics, ITALY

Miss C. De Palo, Associazione EURATOM-ENEA, ITALY

Dr. G. Grosso, Istituto di Fisica del Plasma, ITALY

Prof. G. Rostangni, Istituto Gas lonizzati Del Cnr, ITALY
Dr. H. Yamato, Toshiba Res \& Devel Center, JAPAN

Prof. I. Kawakami, Hiroshima Univ., JAPAN

Prof. K. Nishikawa, Hiroshima Univ., JAPAN

Librarian, Naka Fusion Research Establishment, JAERI, JAPAN

Director, Japan Atomic Energy Research inst., JAPAN

Prot. S. Itoh, Kyushu Univ., JAPAN

Research Info. Ctr., National Instit. for Fusion Science, JAPAN

Prof. S. Tanaka, Kyoto Univ., JAPAN

Library, Kyoto Univ., JAPAN

Prof. N. Inoue, Univ. of Tokyo, JAPAN

Secretary, Plasma Section, Electrotechnical Lab., JAPAN

Dr. O. Mitarai, Kumamoto Inst. of Technology, JAPAN

Dr. G.S. Lee, Korea Basic Sci. Ctr., KOREA

J. Hyeon-Sook, Korea Atomic Energy Research Inst, KOREA

D.I. Choi, The Korea Adv. Inst. of Sci. \& Tech., KOREA

Prof. B.S. Liley, Univ. of Waikato, NEW ZEALAND

Inst of Physics, Chinese Acad Sci PEOPLE'S REP. OF CHINA

Library, Inst. of Plasma Physics, PEOPLE'S REP. OF CHINA

Tsinghua Univ. Library, PEOPLE'S REPUBLIC OF CHINA

Z. Li, S.W. Inst Physics, PEOPLE'S REPUBLIC OF CHINA

Prof. J.A.C. Cabral, Instituto Superior Tecnico, PORTUGAL

Prof. M.A. Hellberg, Univ. of Natal, S. AFRICA

Prof. D.E. Kim, Pohang Inst. of Sci. \& Tech., SO. KOREA

Prot. C.I.E.M.A.T, Fusion Division Library, SPAIN

Dr. L. Stenflo, Univ. of UMEA, SWEDEN

Library, Royal Inst. of Technology, SWEDEN

Prof. H. Wilhelmson, Chalmers Univ. of Tech., SWEDEN

Centre Phys. Des Plasmas, Ecole Polytech, SWITZERLAND

Bibliotheek, Inst. Voor Plasma-Fysica, THE NETHERLANDS

Asst. Prof. Dr. S. Cakir, Middle East Tech. Univ., TURKEY

Dr. V.A. Glukhikh,Sci. Res. Inst. Electrophys.I Apparatus, USSR

Dr. D.D. Ryutov, Siberian Branch of Academy of Sai., USSR

Dr. G.A. Eliseev, I.V. Kurchatov Inst., USSR

Librarian, The Ukr.SSR Academy of Sciences, USSR

Dr. L.M. Kovrizhnykh, Inst. of General Physics, USSR

Kernforschungsanlage GmbH, Zentralbibliothek, W. GERMANY

Bibliothek, Inst. Für Plasmaforschung, W. GERMANY

Prof. K. Schindler, Ruhr-Universitát Boctium, W. GERMANY

Dr. F. Wagner, (ASDEX), Max-Planck-Institut, W. GERMANY

Librarian, Max-Planck-Institut, W. GERMANY 